\title{
Magnon pair emission from a relativistic domain wall in antiferromagnets
}

\author{
Gen Tatara, ${ }^{1}$ Collins Ashu Akosa $\odot,{ }^{1,2}$ and Rubén M. Otxoa de Zuazola ${ }^{3,4}$ \\ ${ }^{1}$ RIKEN Center for Emergent Matter Science (CEMS) and RIKEN Cluster for Pioneering Research (CPR), \\ 2-1 Hirosawa, Wako, Saitama 351-0198, Japan \\ ${ }^{2}$ Department of Theoretical and Applied Physics, African University of Science and Technology (AUST), \\ Km 10 Airport Road, Galadimawa, Abuja FCT, Nigeria \\ ${ }^{3}$ Hitachi Cambridge Laboratory, J. J. Thomson Avenue, CB3 OHE Cambridge, United Kingdom \\ ${ }^{4}$ Donostia International Physics Center, 20018 San Sebastián, Spain
}

(Received 27 July 2020; revised 22 October 2020; accepted 26 October 2020; published 12 November 2020)

\begin{abstract}
Magnon emission and excitation by a relativistic domain wall at a constant velocity in antiferromagnets is theoretically studied. A pair emission due to a quadratic magnon coupling is shown to be dominant. The emission corresponds in the comoving frame to a vacuum polarization induced by a zero-energy instability of the Lorentzboosted anomalous response function. The emission rate is sensitive to the magnon dispersion and wall profile, and is significantly enhanced for a thin wall with velocity close to the effective light velocity. The Ohmic damping constant due to magnon excitation at low velocity is calculated.
\end{abstract}

DOI: 10.1103/PhysRevResearch.2.043226

\section{INTRODUCTION}

Emission from a relativistic moving object is a general intriguing issue that has an analogy to blackbody radiation and black hole physics [1] and can be applied for wave amplification [2]. Solid-state systems are particularly interesting from the viewpoints of quantum effects and experimental feasibility due to low "light velocity." Antiferromagnets at low energy have been known to be typical relativistic systems [3] when dissipation is neglected, and dynamic properties of domain walls have been explained in terms of Lorentz contraction [4].

In this paper, we study the emission from a moving domain wall, a relativistic soliton, in an antiferromagnet. We discuss the low-energy regime using a continuum model, valid when the wall thickness $\lambda$ is larger than the lattice constant $a$. The system is described by a relativistic Lagrangian, and thus there are domain wall solutions moving with a constant velocity smaller than the effective light velocity $c$. The wall width $\lambda$ is affected by Lorentz contraction; $\lambda=\gamma \lambda_{0}$, where $\lambda_{0}$ is the thickness at rest,

$$
\gamma\left(v_{\mathrm{w}}\right) \equiv \sqrt{1-\left(\frac{v_{\mathrm{w}}}{c}\right)^{2}}
$$

is a contraction factor, and $v_{\mathrm{w}}$ is the velocity of the wall.

Emission from a moving object is generally dominated by a linear process, where the object couples to its fluctuation linearly. In the case of soliton solutions, such linear coupling, absent at rest, arises from acceleration and deformation as

Published by the American Physical Society under the terms of the Creative Commons Attribution 4.0 International license. Further distribution of this work must maintain attribution to the author(s) and the published article's title, journal citation, and DOI. argued for ferromagnetic domain walls [5-8]. The dynamics of a magnetic domain wall is described by two collective coordinates, the wall position $X$ and angle $\phi$ of the wall plane. In ferromagnets, $\phi$ is the canonical momentum of the wall, and thus the ferromagnetic wall motion is intrinsically coupled to the angle rotation $[9,10]$. Various origins of the emission have been argued theoretically for a ferromagnetic domain wall (see Ref. [7] and references therein): Couplings of the wall to the second-order magnon field were studied in Refs. [5,7] and a linear coupling to the wall velocity modulation was discussed in Ref. [6]. Modulation of the wall thickness, $\lambda$, was found to contribute to a significant magnon emission numerically [11] and analytically [8]. The emission effect has a practical importance in predicting a damping constant of a wall. Ohmic friction force proportional to the wall velocity was found from the thickness modulation [8], and weaker super-Ohmic frictions were also identified [6,7].

The antiferromagnetic case is expected to be qualitatively distinct from the ferromagnetic case because of a different spin dynamics governed by the Lorentz invariance. The wall coordinates $X$ and $\phi$ are not conjugate to each other, but have independent dynamics (see Appendix A 4). It turns out that the couplings to magnons contain higher-order time derivatives of the wall coordinates compared to the ferromagnetic case, and the linear coupling induces weak super-Ohmic dissipation, negligible at low energy. The dominant emission thus arises from the second-order coupling to the moving wall. A linear momentum is transferred from the wall to magnons, while the energy comes from Doppler shift. In the rest frame of the wall, the wall potential generates a localized magnon excitation. The excitation is described by the normal (particle-hole) component of the magnon response function, which we call $\Pi_{q}$ ( $q$ is the wave vector transferred). In the moving frame, this excitation corresponds to a scattering of magnons, resulting in a significant Ohmic friction force at low velocity. The 
scattering property of the normal response function $\Pi_{q}$ is essentially the same as in the ferromagnetic case studied in Ref. [8]; although the magnon dispersion in ferromagnets, quadratic in the wave vector $k$, is different from the antiferromagnetic linear behavior (in the absence of a gap), it does not lead to qualitative difference in magnon scattering.

A significant feature antiferromagnets have is the existence of an anomalous particle-particle (or hole-hole) propagation, $\Gamma_{q}$, like in superconductivity contributing to the response function [12]. This is due to the quadratic time-derivative term of the relativistic Lagrangian, which allows positive and negative energy (or frequency) equally. The anomalous response function thus can be regarded as a scattering of particles with a positive and negative energies. The negative frequency mode exists generally in any relativistic excitations. In optics, for example, a scattering of negative frequency modes was argued to cause an amplification of photon current [13]. In the context of magnons, the scattering of negative frequency modes corresponds to an emission and absorption of two magnons. The anomalous response function $\Gamma_{q}$ describing such process is shown to be sensitive to the magnon dispersion as well as the wall velocity. Its low-energy weight is much smaller compared to the normal response function $\Pi_{q}$ for the ideally relativistic dispersion of $k$-linear dependence, while it is significantly enhanced if it deviates from linear to have a flatter dispersion. The anomalous response function in this case has a sharp and large peak at finite wave vector for the wall velocity close to the effective light velocity $c$, resulting in a strong forward emission of two magnons. Our results indicate that the relativistic domain wall is useful as a magnon emitter, and the efficiency is tunable by designing magnon dispersion.

A second-order coupling of magnons to a domain wall velocity was studied in the ferromagnetic case in Ref. [7], and it was shown to give rise to a super-Ohmic dissipation. The antiferromagnetic domain wall has been intensively studied recently [4,14-16]. The effect of magnons in driving a wall as a result of the wall potential scattering was theoretically studied in Ref. [14]. At critical wall velocity when the wall width becomes comparable to the lattice spacing, the relativistic nature of the wall breaks down as was discussed in Refs. [16,17].

The pair emission here is analogous to the vacuum polarization (Schwinger pair production of an electron and a positron) by an electric field in vacuum electromagnetism [18]. While in the original context, the effect requires extremely high field and has not been observed so far, solid-state realization by use of graphene has been drawing interest recently [19]. The present magnon emission is an analog of the vacuum polarization in solids, with the role of the electric field played by the moving wall. In fact, in the laboratory frame, the emission occurs when the magnon creation gap of $2 \Delta$ is overcome by the energy shift by the Doppler effect. In the moving frame with the wall, the emission is a spontaneous vacuum polarization induced by a zero-energy instability of the Lorentz-boosted anomalous magnon response function.

\section{MODEL}

Magnetic properties of antiferromagnets are described by the staggered (Néel) order parameter $\boldsymbol{n}$ of the unit length. Its low-energy Lagrangian is relativistic, namely, invariant under the Lorentz transformation as for the kinetic parts [3]. We consider the case with an easy-axis anisotropy energy along the $z$ axis, described by the continuum Lagrangian of

$$
L=\frac{J}{2 a} \int d x\left[\frac{1}{c^{2}} \dot{\boldsymbol{n}}^{2}-(\nabla \boldsymbol{n})^{2}+\frac{1}{\lambda_{0}^{2}}\left(n_{z}\right)^{2}\right],
$$

where $J$ is the exchange energy, and $J / \lambda_{0}^{2}(=K)$ is the easyaxis anisotropy energy. Our results are valid in the presence of hard-axis anisotropy simply by including the effect in the gap of magnons. The effective light velocity is $c=\sqrt{g J}, g$ being a coupling constant [12]. The lattice constant is included to simplify the dimensions of material constants. We consider the one-dimensional case, although the effects we discuss are general and apply to higher-dimensional walls. The Lagrangian is relativistic; i.e., a Lorentz transformation to a moving frame with a constant velocity $v, t^{\prime}=\left(t-\frac{v}{c^{2}} x\right) / \gamma(v)$, and $x^{\prime}=(x-v t) / \gamma(v)$ does not modify the form. The system has a soliton (domain wall) solution, $n_{z}(x)=\tanh \frac{x}{\lambda_{0}}$. The Lorentz invariance indicates moving walls $n_{z}((x-v t) / \gamma)$ are classical solutions for a constant $v<c$, with a contracted thickness $\lambda=\lambda_{0} \gamma(v)$.

These constant-velocity solutions are stable, meaning that they have no linear coupling to magnons and there is no linear emission (see Appendix A). Linear emission may occur during acceleration or by deformation. The emission is studied by introducing collective coordinates [20]. In the case of a domain wall, of most interest is the wall position $X$ [21]. The coupling between the coordinate and fluctuation is governed by the kinetic part of the Lagrangian. In antiferromagnets, it is second order in time derivative, and thus the linear fluctuation $\varphi$ couples to the acceleration $\ddot{X}$ as $\varphi \ddot{X}$ (see Appendix B). The emitted magnon amplitude $\langle\varphi\rangle$ is thus proportional to $\ddot{X}$, and the recoil force on the wall is $\frac{\partial^{2}}{\partial t^{2}}\langle\varphi\rangle \propto \frac{\partial^{4}}{\partial t^{4}} X$. Hence the linear coupling does not induce Ohmic friction and is negligibly small at low energy. The result is the same for other collective variables like thickness oscillation. The motion of an antiferromagnetic domain wall is therefore protected from the damping due to a linear coupling, in contrast to the ferromagnetic case, where Ohmic dissipation arises from thickness oscillation [8].

Instead, emission due to the second-order coupling dominates in antiferromagnets. At low energy, the contribution containing less time derivative of the wall collective coordinates dominates. The issue then reduces to a simple and general problem of the emission from a moving potential of a constant velocity. Our domain wall solution of the tanh profile induces an attractive potential of $\cosh ^{-2}$ form. Taking account of the two magnon modes along the $x$ and $y$ directions, $\varphi_{x}$ and $\varphi_{y}$, respectively $\left[\boldsymbol{n} \simeq\left(\varphi_{x}, \varphi_{y}, 1\right)\right]$, the potential reads (see Appendix A) [20]

$$
V=-K \int \frac{d x}{a} \frac{1}{\cosh ^{2} \frac{x-X(t)}{\lambda}}\left(\varphi_{x}^{2}+\varphi_{y}^{2}\right),
$$

where $X(t)$ is the wall position and $\lambda=\lambda_{0} \gamma$ is the thickness of a moving wall. We consider the case of a constant velocity, $X(t)=v_{\mathrm{w}} t$. A moving potential transfers momentum $q$ to fluctuations and an angular frequency $\Omega$ as a result of the Doppler shift. Although the form of the potential, Eq. (3), is 


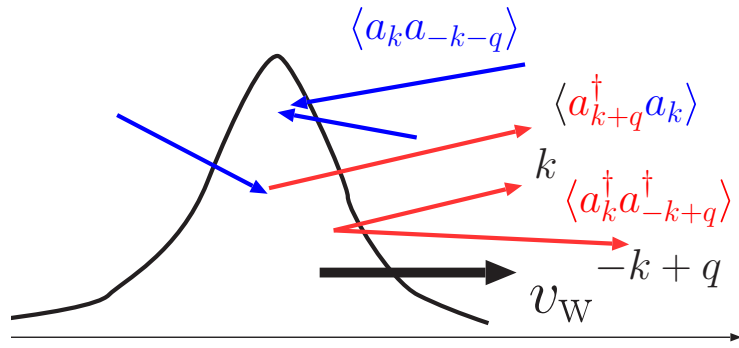

FIG. 1. Schematic figure showing magnon processes due to moving domain wall (black curved line) with a velocity $v_{\mathrm{w}}$; there is a scattering, with the amplitude $\left\langle a^{\dagger} a\right\rangle$, a pair emission $\left(\left\langle a^{\dagger} a^{\dagger}\right\rangle\right)$ and a pair annihilation $(\langle a a\rangle)$. Red and blue arrows denote created and annihilated magnons, respectively. A wave vector $k$ is that of one of the magnons, and $q$ is the transferred wave vector from the wall.

common for ferro- and antiferromagnetic cases, its effect is different, due to the different nature of magnon excitations. In ferromagnets, $\varphi_{x}$ and $\varphi_{y}$ are represented as linear combinations of magnon fields $b$ and $b^{\dagger}$ (Holstein-Primakov boson). The potential in this case is proportional to magnon density as $\varphi_{x}^{2}+\varphi_{y}^{2}=4 b^{\dagger} b$, inducing scattering of magnons without changing total magnon number. (The feature is unchanged in the presence of a hard-axis anisotropy.) This is due to the kinetic term linear in the time derivative for ferromagnetic magnons [20], $i b^{\dagger} \dot{b}$, which allows a positive energy for the ferromagnetic magnon boson. In contrast, a magnon boson in antiferromagnets is described by a relativistic Lagrangian (see Appendix A)

$$
L_{\varphi}=\frac{1}{2 g} \sum_{i, k}\left\{\left[\dot{\varphi}_{i}(k)\right]^{2}-\left(\omega_{k}^{(i)}\right)^{2}\left[\varphi_{i}(k)\right]^{2}\right\},
$$

where $i=x, y$ and

$$
\omega_{k}^{(i)} \equiv \sqrt{c^{2} k^{2}+\Delta_{i}^{2}}
$$

is the energy with a gap $\Delta_{i}$ of mode $i$. The kinetic term second order in time derivative, $\left(\dot{\varphi}_{i}\right)^{2}$, allows "negative frequency" modes, and processes changing the total magnon number are allowed. In fact, the canonical magnon boson $a_{i}$ is defined for each mode $i=x, y$ as

$$
\varphi_{i}(k, t)=\sqrt{\frac{g}{\omega_{k}^{(i)}}}\left[a_{k}^{(i)}(t)+a_{-k}^{(i) \dagger}(t)\right],
$$

and the potential, Eq. (3), reads

$$
\begin{aligned}
V= & -K g \frac{\lambda}{a} \sum_{i=x, y} \sum_{k, q} \frac{W_{q}}{\sqrt{\omega_{k}^{(i)} \omega_{k+q}^{(i)}}} e^{-i q X(t)} \\
& \times\left(a_{k}^{(i)} a_{-k-q}^{(i)}+a_{-k}^{(i) \dagger} a_{k+q}^{(i) \dagger}+2 a_{k+q}^{(i) \dagger} a_{k}^{(i)}\right),
\end{aligned}
$$

where $W_{q}=\pi \frac{q \lambda}{\sinh \frac{\pi}{2} q \lambda}$ is the Fourier transform of the potential profile and $\omega_{-k}=\omega_{k}$ is assumed. The emission and absorption of two magnons, represented by terms $a a$ and $a^{\dagger} a^{\dagger}$, are thus possible in antiferromagnets (Fig. 1).

The potential represents a transfer of linear momentum between the wall and magnons, while angular momentum is not transferred. This is because the angular momentum, $\ell$, of antiferromagnets is represented by a product of $\varphi_{x}$ and $\varphi_{y}$ as $\ell=-\frac{1}{2 S g}(\boldsymbol{\varphi} \times \dot{\boldsymbol{\varphi}})(S$ is the magnitude of localized spin) [12]. Although the potential contains pair creation and annihilation processes within a single magnon branch, it does not therefore change the angular momentum of magnons.

The relativistic nature is partially lost when dissipation is included. We here consider the case of weak dissipation (small Gilbert damping).

\section{MAGNON AMPLITUDES}

Let us evaluate the amplitudes of scattering and emission and absorption as a linear response to the dynamic potential $V$ for a wall moving with a constant velocity. We suppress the index $i$ for the magnon branch. The scattering amplitude,

$$
\left\langle a_{k+q}^{\dagger} a_{k}\right\rangle(t)=i G_{k, k+q}^{<}(t, t),
$$

is a lesser Green's function for magnons. The amplitude after summation over $k$ is represented in terms of the normal (particle-hole) response function, including the form factor $W_{q}$ $[8,12]$ (see Appendix C),

$$
\Pi_{q, \Omega} \equiv-\sum_{k} \frac{W_{q}}{\sqrt{\omega_{k} \omega_{k+q}}} \frac{n_{k+q}-n_{k}}{\omega_{k+q}-\omega_{k}-\Omega+2 i \eta},
$$

as

$$
\sum_{k}\left\langle a_{k+q}^{\dagger} a_{k}\right\rangle=\frac{K g}{a} \lambda e^{i q v_{\mathrm{w}} t} \Pi_{q},
$$

where $\Pi_{q} \equiv \Pi_{q, q v_{\mathrm{w}}}$. Here $n_{k} \equiv\left[e^{\beta \omega_{k}}-1\right]^{-1}$ is the Bose distribution function, $\beta \equiv\left(k_{B} T\right)^{-1}$ being the inverse temperature ( $k_{B}$ is the Boltzmann constant); $\eta$ is the damping coefficient of the magnon Green's function. The angular frequency of $\Omega=q v_{\mathrm{w}}$ in $\Pi_{q, q v_{\mathrm{w}}}$ is the one transferred to magnons as a result of the Doppler shift. The emission amplitude of two magnons is

$$
\sum_{k}\left\langle a_{-k+q}^{\dagger} a_{k}^{\dagger}\right\rangle=\frac{K g}{a} \lambda e^{i q v_{\mathrm{w}} t} \Gamma_{q},
$$

where $\Gamma_{q} \equiv \Gamma_{q, q v_{\mathrm{w}}}$ and

$$
\Gamma_{q, \Omega} \equiv \sum_{k} \frac{W_{q}}{\sqrt{\omega_{k} \omega_{-k+q}}} \frac{1+n_{-k}+n_{-k+q}}{\omega_{-k+q}+\omega_{k}-\Omega+2 i \eta}
$$

is the anomalous (particle-particle) response function. The absorption amplitude is given by this function as ${ }^{*}$ denotes the complex conjugate)

$$
\sum_{k}\left\langle a_{-k+q} a_{k}\right\rangle=\frac{K g}{a} \lambda e^{-i q v_{\mathrm{w}} t} \Gamma_{-q}^{*} .
$$

The normal response function has symmetry of $\Pi_{-q, \Omega}=$ $\Pi_{q, \Omega}$, which leads in the case of $\Omega=q v_{\mathrm{w}}$ to $\Pi_{-q,-q v_{\mathrm{w}}}=$ $\Pi_{q, q v_{\mathrm{w}}}^{*}$; i.e., the real (imaginary) part of $\Pi_{q}$ is even (odd) in $q$. The normal response has a low-energy contribution around $q=0$ and $\Omega=0$. The asymmetric and localized character near $q=0$ of $\operatorname{Im}\left[\Pi_{q}\right]$ indicates an asymmetric real-space magnon distribution with respect to the wall center similarly to the ferromagnetic case [8]. The anomalous response satisfies $\Gamma_{-q, \Omega}=\Gamma_{q, \Omega}$. It has a gap of $2 \Delta$ for $\Omega$, suppressing 


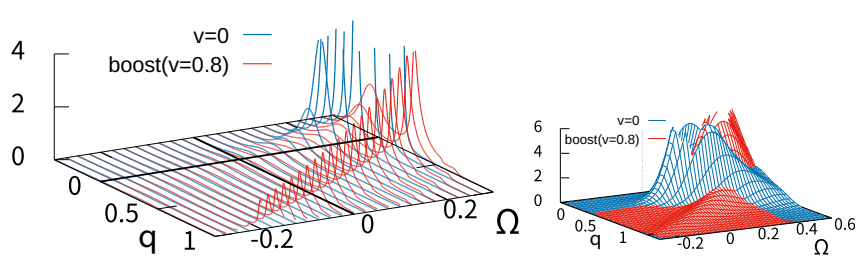

FIG. 2. Effect of Lorentz boost on the anomalous response function $\operatorname{Im}\left[\Gamma_{q, \Omega}\right]$ at $v_{\mathrm{w}} / c=0.8$ for a hyperbolic dispersion $(\mu=5)$, $\tilde{\lambda_{0}}=2, \tilde{\Delta}=0.1, \tilde{\eta}=0.01$, and $\tilde{T}=0.2$ in dimensionless arbitrary units. Blue is the amplitude at rest frame, which is localized at $\Omega \gtrsim 2 \Delta$ with negligibly small weight at $\Omega=0$. In the boosted frame, shown in red, the amplitude extends to the zero-energy regime at finite $q$, inducing spontaneous vacuum polarization, which corresponds to a pair emission in the laboratory frame. The right plot is a global plot.

the low-energy contribution in the rest frame (Fig. 2). In the moving frame, the Lorentz boost, which transforms $q$ and $\Omega$ to

$$
q^{\prime}=\left(q+v_{\mathrm{w}} \Omega / c^{2}\right) / \gamma, \quad \Omega^{\prime}=\left(\Omega+v_{\mathrm{w}} q\right) / \gamma,
$$

distorts the response function, enhancing significantly the low-energy weights at finite $q$ (Fig. 2 and Appendix D). This induces spontaneous vacuum polarization, which corresponds to a two-magnon emission in the laboratory frame.

There are two key factors governing the response functions, the form factor $W_{q}$ and magnon dispersion. The form factor constrains the wave vector transfer $q$ to $|q| \lesssim \lambda^{-1}=$ $\left(\lambda_{0} \gamma\right)^{-1}$. Because of this factor, magnon effects are significantly enhanced for thin walls at high velocity ( $\operatorname{small} \gamma$ ). As the emission is dominated by the large- $q$ behavior, it is sensitive to the wall profile as we shall see below.

The role of the dispersion is clearly seen focusing on the imaginary part in the limit of $\eta \rightarrow 0$, where the response arises from the processes satisfying the energy and momentum conservation. We consider the case of the dispersion with a small gap and saturation around $k_{\max }=\pi / a$, like the one in $\mathrm{MnF}_{2}$ [22]. We choose $v_{\mathrm{w}}$ as positive. The imaginary part of the normal response arises from the process satisfying $\omega_{k+q}-\omega_{k}=q v_{\mathrm{w}}$ [Fig. 3(a)], which leads to an asymmetric weight around $q=0$. The imaginary part of the anomalous response $\Gamma_{q}$ arises when

$$
\omega_{-k+q}+\omega_{k}=q v_{\mathrm{w}}
$$

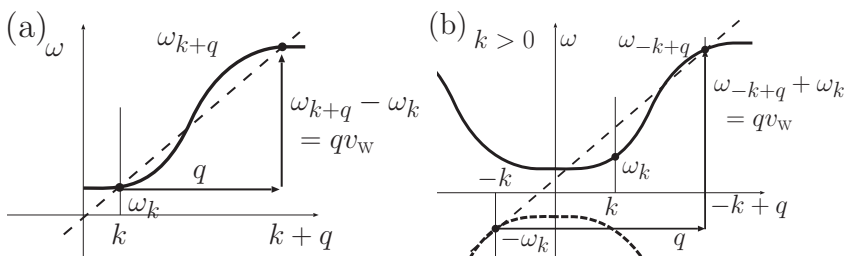

FIG. 3. Energy conservation conditions in (a) scattering and (b) emission and absorption of two magnons. The emission is regarded as a scattering from a hole state with energy $-\omega_{k}$ to a particle state with energy $\omega_{-k+q}$. The slope of dotted straight lines is $v_{\mathrm{w}}$.
This amplitude is much smaller than the normal response for the relativistic dispersion, $\omega_{k}=\sqrt{(c k)^{2}+\Delta^{2}}$, due to the following reasons [Fig. 3(b)]: The process satisfying Eq. (15) is regarded as a scattering process of a particle and a hole having positive and negative energy, $\omega_{-k+q}$ and $-\omega_{k}$, respectively. The condition requires that the average slope of the line connecting the two energies $\omega_{-k+q}$ and $-\omega_{k}$ is $v_{\mathrm{w}}$. However, the slope is larger than $c$ for the relativistic dispersion, while $v_{\mathrm{w}}$ has an upper limit of $c$, which is the maximum group velocity. The condition cannot therefore be satisfied by the purely relativistic dispersion, and the imaginary part of the anomalous response thus arises only if the dispersion has an inflection point like in Fig. 3(b). (In reality, a damping $\eta$ leads to a finite imaginary part, but it remains negligibly small.) Those features are consistent with a theory of spin transport in antiferromagnets [12] showing that the anomalous correlation function is negligible.

\section{NUMERICAL RESULTS}

Let us evaluate numerically the response functions of scattering and emission and absorption for a domain wall at constant velocity. We use dimensionless variables, defined by scaling by $k_{\max }=\pi / a$ for wave vectors and by $\pi c / a$ for energies ( $\hbar$ is set to unity),

$$
\begin{aligned}
& \tilde{q}=q / k_{\max }, \quad \tilde{k}=k / k_{\max }, \quad \tilde{\omega}_{k}=\frac{a}{\pi c} \omega_{k}, \\
& \tilde{v}=V_{\mathrm{w}} / c, \quad \tilde{\lambda_{0}}=\frac{\lambda_{0}}{a}, \quad \tilde{t}=\frac{\pi c}{a} t, \\
& \tilde{\Delta}=\frac{a}{\pi c} \Delta, \quad \tilde{\eta}=\frac{a}{\pi c} 2 \eta, \quad \tilde{T}=\frac{a}{\pi c} k_{B} T .
\end{aligned}
$$

We have also $K g=\frac{c^{2}}{\lambda_{0}^{2}}$.

The amplitudes we calculate are

$$
\begin{aligned}
\left\langle a_{k+q}^{(i) \dagger} a_{k}^{(i)}\right\rangle & =\frac{1}{\pi^{2}} \frac{\lambda a}{\lambda_{0}^{2}} e^{i \tilde{q} \tilde{v} \tilde{t}} \tilde{\Pi}_{k q}, \\
\left\langle a_{-k+q}^{(i) \dagger} a_{k}^{(i) \dagger}\right\rangle & =\frac{1}{\pi^{2}} \frac{\lambda a}{\lambda_{0}^{2}} e^{i \tilde{q} \tilde{v} \tilde{t}} \tilde{\Gamma}_{k q},
\end{aligned}
$$

where

$$
\begin{aligned}
\tilde{\Pi}_{k q} & \equiv-\frac{W_{-q}}{\sqrt{\tilde{\omega}_{k}^{(i)} \tilde{\omega}_{k+q}^{(i)}}} \frac{n_{k+q}-n_{k}}{\tilde{\omega}_{k+q}^{(i)}-\tilde{\omega}_{k}^{(i)}-\tilde{q} \tilde{v}+i \tilde{\eta}}, \\
\tilde{\Gamma}_{k q} & \equiv \frac{W_{-q}}{\sqrt{\tilde{\omega}_{k}^{(i)} \tilde{\omega}_{-k+q}^{(i)}}} \frac{1+n_{k}+n_{-k+q}}{\tilde{\omega}_{-k+q}^{(i)}+\tilde{\omega}_{k}^{(i)}-\tilde{q} \tilde{v}+i \tilde{\eta}}
\end{aligned}
$$

are dimensionless response functions. The amplitudes after $k$ summation are defined as

$$
\tilde{\Pi}_{q} \equiv \sum_{k} \tilde{\Pi}_{k q}, \quad \tilde{\Gamma}_{q} \equiv \sum_{k} \tilde{\Gamma}_{k q}
$$

\section{A. Hyperbolic dispersion}

As Fig. 3(b) suggests, the anomalous emission is enhanced for a band with smaller average slope keeping the maximum slope (the maximum group velocity) as $c$. We take here as an 


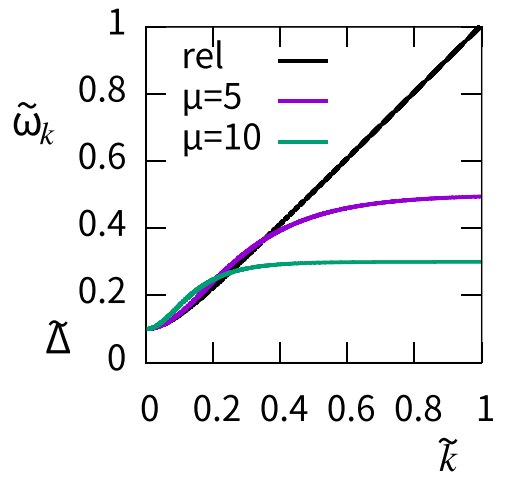

FIG. 4. Dispersions considered: relativistic (rel; black line) and hyperbolic dispersions with $\mu=5,10$. The maximum slope (maximum group velocity) is $c(=1)$ and the gap is $\tilde{\Delta}=0.1$ for both cases.

example a hyperbolic form of

$$
\omega_{k}^{(\mathrm{h})}=\Delta+\frac{2 c k_{\max }}{\mu}\left(1-\frac{1}{\cosh \mu k / k_{\max }}\right),
$$

where $k_{\max }=\pi / a$ and $\mu$ is a parameter defining the average slope [23]. The two dispersions,

$$
\begin{array}{ll}
\tilde{\omega}_{k}=\sqrt{\tilde{k}^{2}+\tilde{\Delta}^{2}} & \text { (relativistic dispersion), } \\
\tilde{\omega}_{k}=\tilde{\Delta}+\frac{2}{\mu}\left(1-\frac{1}{\cosh \mu \tilde{k}}\right) & \text { (hyperbolic dispersion), }
\end{array}
$$

are plotted in Fig. 4.

\section{B. Wall profiles}

The anomalous emission, determined by the large- $q$ behavior of the response function, is sensitive to the wall profile. In the case of a very thin wall, the linear profile of $n_{x}$ (or $n_{y}$ ) inside the wall may appear instead of the ideal tanh wall, as argued in nanocontacts [24], which is

$$
n_{x}=\left(1-|x| / \lambda^{(1)}\right) \theta\left(\lambda^{(1)}-|x|\right),
$$

where $\lambda^{(1)}=\lambda \pi / 2$. The two wall profiles and form factors,

$$
W_{q} \equiv\left\{\begin{array}{lr}
\frac{\pi^{2} \tilde{\lambda} \tilde{q}}{\sinh \frac{\pi^{2}}{2} \tilde{\lambda} \tilde{q}} & \text { (tanh wall), } \\
\frac{2 \pi}{\left(\tilde{\lambda}^{(1)} \tilde{q}\right)^{2}}\left(1-\frac{\sin \tilde{\lambda}^{(1)} \tilde{q}}{\tilde{\lambda}^{(1)} \tilde{q}}\right) & \text { (linear wall) }
\end{array}\right.
$$

with $\tilde{\lambda}=\lambda / a$ and $\tilde{\lambda}^{(1)}=\frac{\pi}{2} \lambda / a$ are plotted in Fig. 5 .

$$
n_{x}=\left\{\begin{array}{lr}
\frac{1}{\cosh \frac{x}{\lambda}} & \text { (tanh wall), } \\
\left(1-|x| / \lambda^{(1)}\right) \theta\left(\lambda^{(1)}-|x|\right) & \text { (linear wall). }
\end{array}\right.
$$

\section{Response functions}

Let us first show the most typical effect of wall motion on the imaginary part of the anomalous response function, $\operatorname{Im}\left[\Gamma_{q}\right]$ [Fig. 6(a)]. For a relativistic dispersion, $\operatorname{Im}\left[\Gamma_{q}\right]$ has only a low peak near $q=0$, while for hyperbolic dispersion it is significantly altered; a sharp peak appears for velocity $v_{\mathrm{w}} / c \gtrsim 0.7$ at $q=q^{*}$ in the high- $q$ regime $\left(0.5 \lesssim q^{*} / k_{\max } \lesssim\right.$ $1)$, indicating strong forward emission of two magnons. The
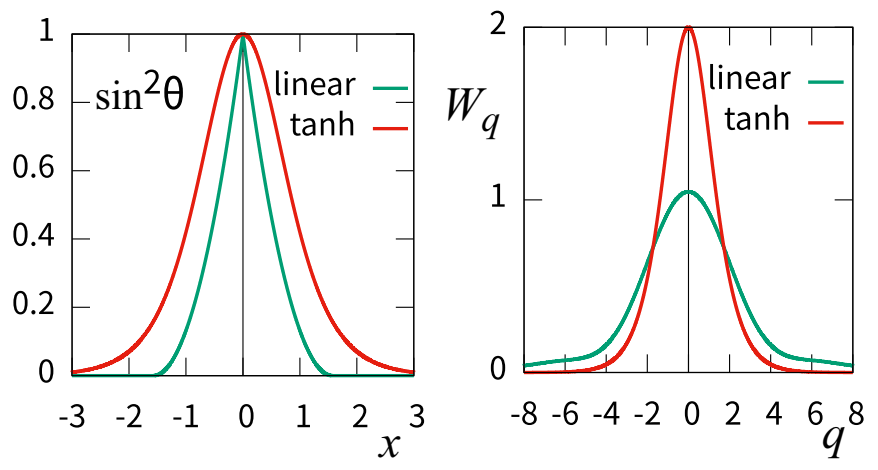

FIG. 5. Wall profiles, $\sin ^{2} \theta(x)$, for tanh and linear walls, and their Fourier transforms (form factors) $W_{q}$. Linear wall has slow power-law decay at large $q$, resulting in the enhancement of the anomalous response function (see Figs. 6 and 9).

minimum velocity necessary is determined by the dispersion; it is obviously larger than $\omega_{k_{\max }} / k_{\max }$ for a monotonically increasing dispersion, which is $\sim \frac{2}{\mu} c$ for hyperbolic dispersion with a small gap. These behaviors are consistent with the argument in Sec. III. The peak position $q^{*}$ is independent of $\lambda_{0}$. The intensity $I^{*}$ of the peak and $q^{*}$ are plotted as functions of velocity in Fig. 6(b). The anomalous response amplitude $I^{*}$ is sensitive to the wall profile; it is significantly enhanced for a linear wall due to a slower decay at large $q$ compared to the tanh wall [Fig. 6(b)].

The present continuum approximation breaks down for $\lambda \lesssim a$, i.e., for $v \gtrsim v_{c} \equiv \sqrt{1-\left(a / \lambda_{0}\right)^{2}}$. For $\lambda_{0} / a=2,4,6,8$, the critical velocity is $v_{c} / c=0.866,0.968,0.986,0.992$, respectively, as shown by the vertical dotted lines in Fig. 6(b).

Let us look into the details of the response functions. The real and the imaginary parts of the normal and the anomalous response functions are plotted in Fig. 7 for the case of $\tilde{\lambda}_{0}=2$ at $\tilde{T}=0.8$ for the relativistic and hyperbolic dispersions.

a. Relativistic dispersion. It is seen that the normal response function $\Pi_{q}$ satisfies $\Pi_{-q}=\Pi_{q}^{*}$ and has the real and imaginary part even and odd in $q$, respectively. The wall velocity distorts the profile, but qualitative feature is untouched. The anomalous response function $\Gamma_{q}$ has a negligibly small imaginary part in the case of relativistic dispersion, consistent with the observation in Sec. III and Fig. 2. The peak at $q=0$ of $\operatorname{Re} \Gamma_{q}$ is shifted to the positive- $q$ regime in the presence of positive $v_{\mathrm{w}}$.

b. Hyperbolic dispersion. For hyperbolic dispersion (Fig. 7, lower panel), $\Pi_{q}$ is distorted but the qualitative feature is unchanged. In contrast, $\operatorname{Im} \Gamma_{q}$ is significantly modified at high wall velocity; it shows a sharp peak at finite $q$, as a result of pair creation (annihilation) processes.

c. Thickness dependence. Comparison of $\tilde{\lambda}_{0}=2$ and 4 is shown in Fig. 8. For a thicker wall with $\tilde{\lambda}_{0}=4$, the amplitudes of $\Pi_{q}$ and $\Gamma_{q}$ are reduced and the peak of $\operatorname{Im} \Gamma_{q}$ is shifted to higher wall velocity.

d. Wall-profile dependence. The details of the peak of $\operatorname{Im} \Gamma_{q}$ are shown in Fig. 9 for the tanh and linear profile of the wall. It is seen that the linear profile with slower decay at large $q$ has larger response, although both have qualitatively similar behaviors. 

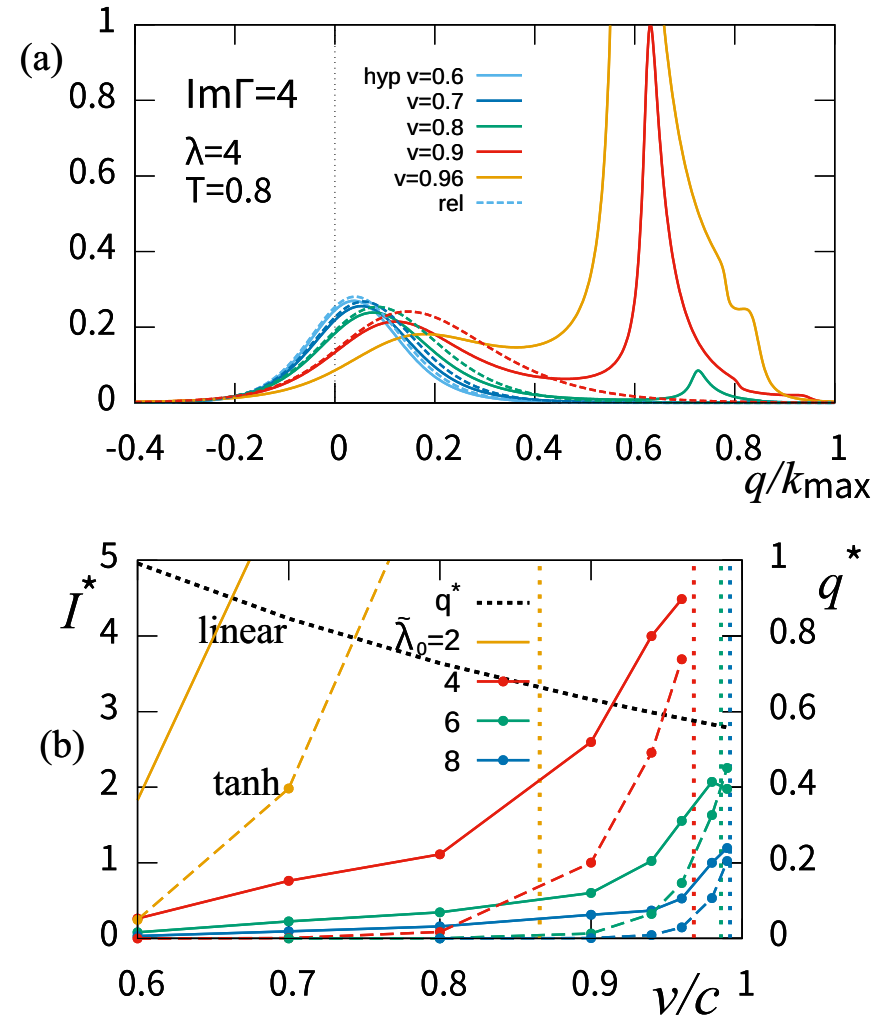

FIG. 6. (a) $\operatorname{Im} \Gamma_{q}$ for relativistic (dotted lines) and hyperbolic (solid lines) dispersion for $v_{\mathrm{w}} / c=0.6,0.7,0.8,0.96$ with $\mu=5$ and $\lambda_{0} / a=4$. At high velocity the hyperbolic dispersion shows a sharp peak at finite $q\left(\equiv q^{*}\right)$, while the response function for the relativistic dispersion, with low and broad peak near $q=0$, is not strongly affected by the velocity. (b) The peak amplitude $I^{*}$ and position $q^{*}$ (black dotted line) of $\operatorname{Im} \Gamma_{q}$ for different $\lambda_{0} / a$. Wall profiles are linear (solid lines) and tanh (dashed lines). The linear wall has larger amplitudes due to a slower decay of $W_{q}$ at large wave vector $q$. The breakdown velocities $v_{c}$ of the continuum approximation are shown as vertical dotted lines.

\section{Magnon current}

The amplitude of the emitted magnon current is estimated by

$$
j=\sum_{k} q\left\langle a_{-k+q}^{\dagger} a_{k}^{\dagger}\right\rangle \sim q^{*} I^{*} .
$$

For $\lambda_{0} / a=4, j \gtrsim 0.8$ for $v_{\mathrm{w}} / c>0.8$, and $j=0.2$ for $\lambda_{0} / a=8$ at $v_{\mathrm{w}} / c=0.9$, at $T=0.8$ and for a linear wall profile. Let us compare the emitted spin wave current with the current due to the wall motion. The magnon current is defined in terms of the real spin wave field $\varphi$ as

$$
j=-\frac{i}{2} a^{\dagger} \stackrel{\leftrightarrow}{\nabla} a=-\frac{1}{4 g}(\dot{\varphi} \stackrel{\leftrightarrow}{\nabla} \varphi)
$$

For a domain wall with the tanh profile, $\varphi^{\mathrm{w}}=\left[\cosh \frac{x-X(t)}{\lambda}\right]^{-1}$. The current at the wall center is thus $j^{\mathrm{w}} \equiv \frac{V_{\mathrm{w}}}{4 g \lambda^{2}}$. Using $J / a^{2} \simeq$ $c / a$, we have

$$
j^{\mathrm{w}} \simeq \frac{k_{\max }}{4 \pi \tilde{\lambda}_{0}^{2}} \frac{\tilde{v}}{1-\tilde{v}^{2}}
$$
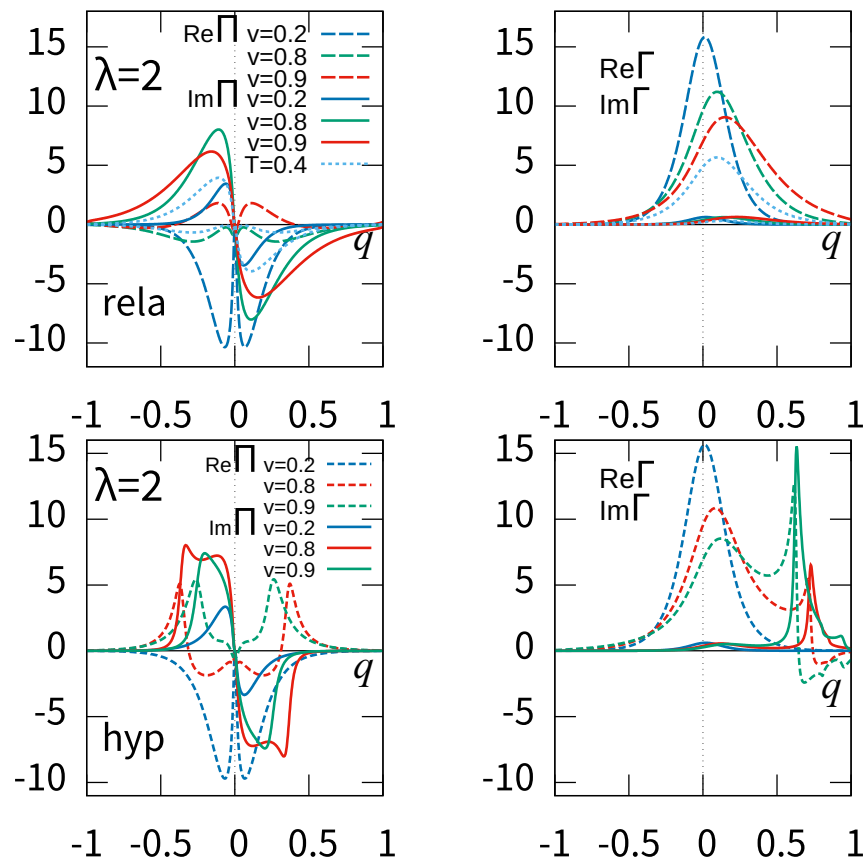

FIG. 7. Plot of the real and the imaginary parts of response functions $\Pi_{q}$ and $\Gamma_{q}$ for relativistic (rela) dispersion and a hyperbolic (hyp) dispersion with $\mu=5$. Calculated for $v_{\mathrm{w}} / c=0.2,0.8,0.9$ at $\tilde{T}=0.8$ with $\tilde{\lambda}\left(\equiv \lambda_{0} / a\right)=2$ and $\tilde{\Delta}=0.1, \tilde{\eta}=10^{-2}$. The effect of temperature is shown for relativistic dispersion at $\tilde{v}=0.8$. The amplitude is reduced by about a factor of 2 at lower temperature of $\tilde{T}=0.4$ compared to $\tilde{T}=0.8$.

where $\tilde{v}=v_{\mathrm{w}} / c$. For $\tilde{\lambda}=4, j^{\mathrm{w}} / k_{\max } \simeq 0.01(0.02)$ at $\tilde{v}=$ $0.8(0.9)$. The current due to the emission is thus by $1-2$ orders of magnitude larger than the current of the wall itself in the relativistic regime. A thin and relativistic wall is therefore an extremely efficient magnon emitter.

\section{E. Recoil force and Gilbert damping parameter}

As a reaction to the scattering, emission and absorption, a frictional force,

$$
F=2 \frac{K g}{a} \operatorname{Im} \sum_{k, q} \frac{W_{q} e^{-i q X(t)}}{\sqrt{\omega_{k} \omega_{k+q}}} q \lambda\left\langle a_{k+q}^{\dagger} a_{k}+a_{-k}^{\dagger} a_{k+q}^{\dagger}\right\rangle,
$$

arises. As seen in the plot of Fig. 10, the emission contribution has a narrow peak at high velocity close to $v_{\mathrm{w}} / c=1$, while the normal-response $(\Pi)$ contribution shows a broad peak starting from the low-velocity regime. The normal contribution is larger than the emission contribution as the excited magnon profile is mostly localized near the wall, resulting in a large overlap. The force at small velocity, dominated by the normal response, is an Ohmic friction,

$$
F=-\alpha v_{\mathrm{w}} / \lambda^{2}
$$

whose dimensionless coefficient $\alpha$ is plotted in Fig. 10. As the force arises from transfer of finite $q$, the friction constant $\alpha$ depends strongly on the wall thickness. The friction coefficient $\alpha$ corresponds to a contribution to the Gilbert damping 

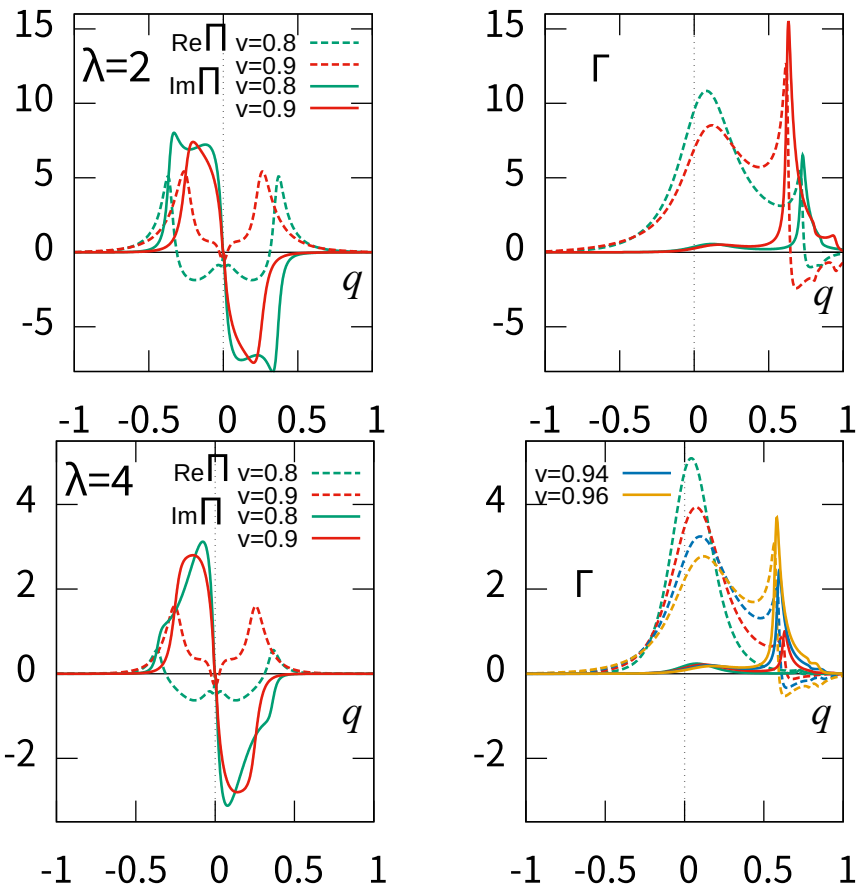

$\begin{array}{lllll}-1 & -0.5 & 0 & 0.5 & 1\end{array}$

FIG. 8. Comparison of $\Pi_{q}$ and $\Gamma_{q}$ for $\tilde{\lambda}_{0}=2$ and 4 for hyperbolic dispersion with $\mu=5$.

constant of

$$
\alpha_{G}=\frac{a}{2 \lambda} \alpha,
$$

which is plotted by dashed lines. For a linear wall profile, the contribution $\alpha_{\mathrm{G}}$ is $0.007(0.002)$ for $\lambda / a=6(8)$ at $T=0.8$, which is significantly large compared to the intrinsic Gilbert damping constant of most antiferromagnets. The damping due to magnon excitation has clear temperature dependence, exponentially suppressed for $k_{B} T \lesssim \Delta$, and increases linearly at high temperature below the Néel transition temperature. For quantitative study, the temperature dependence of $\eta$ and the fluctuation near the Néel temperature need to be taken into account [12].

The temperature dependence of the recoil force is shown in Fig. 11. The slope, the friction constant $\alpha$, and thus the contribution to the Gilbert damping constant, increases nearly linearly for $\tilde{T}>\tilde{\Delta}$. Near the Néel transition, this estimate is insufficient as it does not include the temperature-dependent
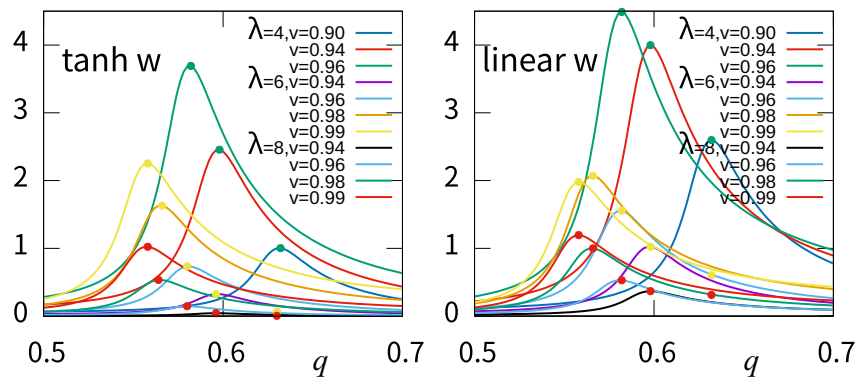

FIG. 9. Comparison of $\operatorname{Im} \Gamma_{q}$ for the wall profiles $\tanh (\tanh$ w) and linear (linear w) walls for $\tilde{\lambda}_{0}=4,6,8$ and for hyperbolic dispersion with $\mu=5$. Peak positions are shown by small circles.
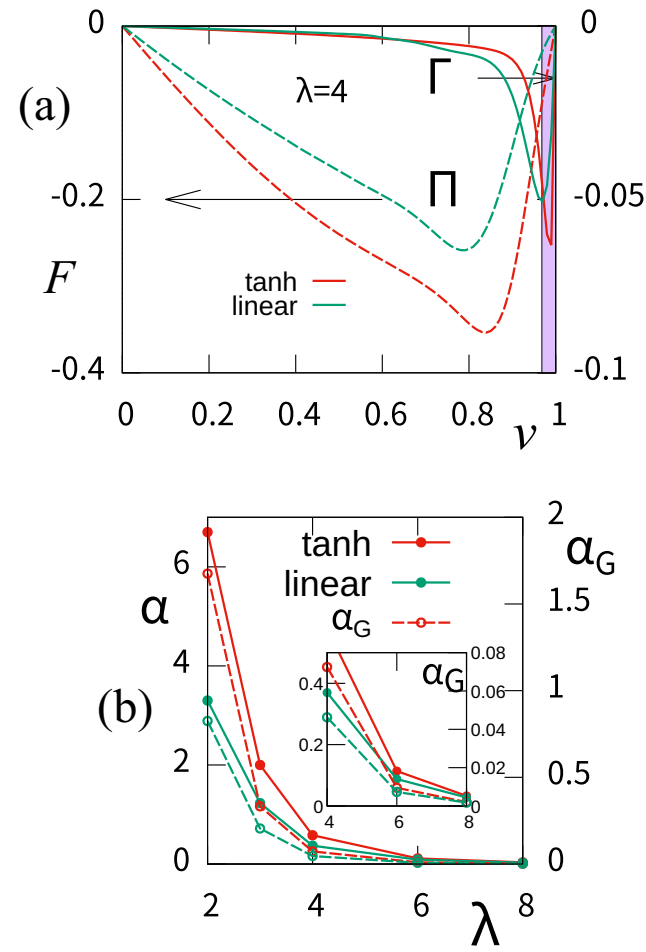

FIG. 10. (a) Plot of the force $F$ as functions of wall velocity for tanh and linear wall with $\lambda_{0} / a=4$. The normal ( $\left.\Pi\right)$ and anomalous $(\Gamma)$ contributions to the force are shown by dashed and solid lines with axis at the left and right, respectively. Shaded region $(\tilde{v}>0.97)$ shows a breakdown of continuum description, i.e., $\lambda \lesssim a$, for $\lambda_{0} / a=$ 4. (b) Friction constant $\alpha$ (left axis) and contribution to the Gilbert damping constant (right axis) determined at small wall velocity $(\tilde{v} \sim$ $0)$ as a function of wall thickness.

damping constant $\eta$ and suppression of the order parameter argued in Ref. [12].

\section{F. Group velocity}

The excited magnon propagates to the direction determined by the sign of its wave vector $k$. As the wall is moving, however, even a forward-emitted magnon may be behind the wall if its group velocity is less than the wall velocity. Figure 12 is a plot of the normal response function for the relativistic

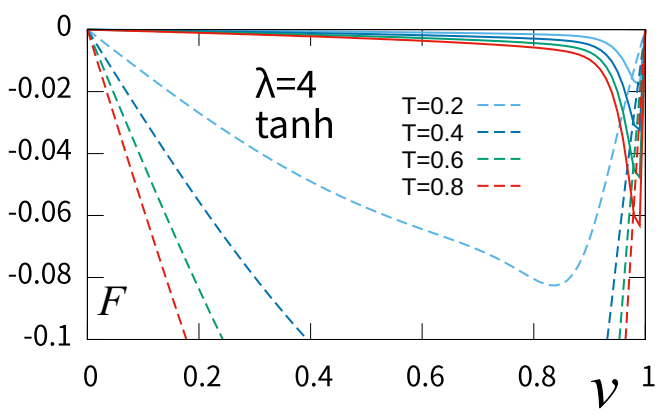

FIG. 11. Plot of the force $F$ for tanh wall with $\lambda_{0} / a=4$ for different temperatures. The normal $(\Pi)$ and anomalous $(\Gamma)$ contributions to the force are shown by dashed and solid lines, respectively. 

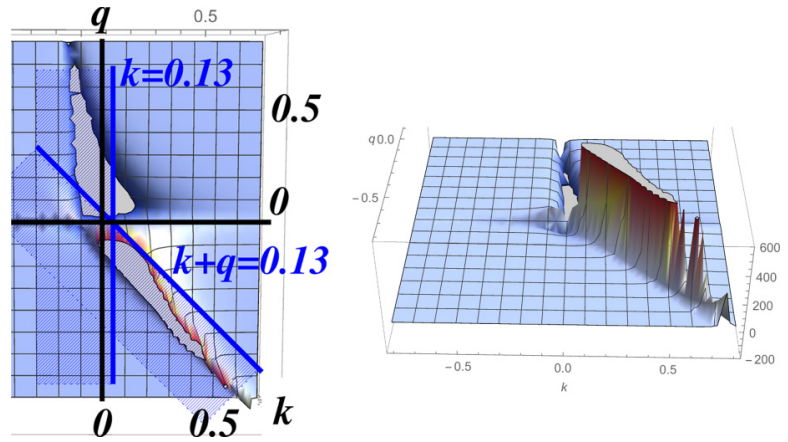

FIG. 12. Normal response function at $v_{\mathrm{w}} / c=0.8$ plotted in the $k$ - $q$ plane for $\tilde{\lambda}_{0}=2, \tilde{\Delta}=0.1, \tilde{\eta}=0.01$, and $\tilde{T}=0.8$. Blue straight lines, $k=0.13$ and $k+q=0.13$, represent the wave vectors $k$ and $k+q$, respectively, where the group velocity $v_{\mathrm{g}}(k)=\frac{\partial \omega_{k}}{\partial k}$ is equal to the wall speed, 0.8. Shaded regime below those lines is therefore magnon excitations behind the wall.

dispersion plotted in the $k-q$ plane. In the case of the wall velocity $v_{\mathrm{w}} / c=0.8$, the wave vector $k^{*}$ where the group velocity is equal to the wall velocity is $k^{*}=0.13$ if $\tilde{\Delta}=0.1$. The excited magnon with wave vector $k+q$ is therefore behind the wall and is $k+q<0.13$. As seen from Fig. 12, the dominant part of the excitation due to the normal response function is behind the wall. This result is consistent with the observation based on the Landau-Lifshitz-Gilbert (LLG) equation analysis in Ref. [4] that the moving wall emits magnons mostly behind the wall. The LLG study fixes the magnon dispersion to be relativistic, and thus its results are due to the normal response function of the present analysis.

\section{SUMMARY}

We have studied theoretically the magnon scattering and emission and absorption due to a moving domain wall in an antiferromagnet. The emission and absorption due to linear couplings to magnons are negligible at low energy as a result of the relativistic nature of antiferromagnetic magnons. Instead, the second-order coupling to the potential induced by the wall is the dominant process, and the scattering and the emission and absorption properties are represented by the normal (particle-hole) and anomalous (particle-particle) response functions, respectively. For a moving wall, the anomalous response function turns out to be significantly deformed due to the Lorentz boost, resulting in a strong emission in the relativistic regime.

As the amplitude $\left\langle a_{-k+q}^{\dagger} a_{k}^{\dagger}\right\rangle$ indicates, the two-magnon pair created by the mechanism proposed here is entangled quantum mechanically like in the case of electromagnetism [25], suggesting interesting possibilities for quantum magnonics.

\section{ACKNOWLEDGMENTS}

This work was supported by a Grant-in-Aid for Scientific Research (B) (Grant No. 17H02929) from the Japan Society for the Promotion of Science.

\section{APPENDIX A: MAGNON LAGRANGIAN}

\section{Rotated frame}

Here we show the derivation of the magnon (spin wave) Lagrangian including the linear and the second-order couplings to the wall from the spin Lagrangian, Eq. (2). We use a transformation to a rotated frame to represent magnon excitations around a domain wall solution. Instead of using collective coordinates for the wall from the start like in Ref. [8], we carry out the calculation generally using the polar angles to describe a classical solution, $\left(\theta_{0}(x, t), \phi_{0}(x, t)\right)$. For a moving domain wall solution of a constant velocity $v_{\mathrm{w}}(\leqslant c)$,

$$
\cos \theta_{0}=\tanh \frac{x-v_{\mathrm{w}} t}{\lambda}, \quad \phi_{0}=0
$$

with a contracted width

$$
\lambda=\lambda_{0} \sqrt{1-\frac{v_{\mathrm{w}}^{2}}{c^{2}}} .
$$

The spin wave is defined in the locally rotated frame, where the quantization axis is chosen along the local direction of the Néel vector, $\left(\theta_{0}(x, t), \phi_{0}(x, t)\right)$. We write the transverse fluctuations as $\varphi_{x}$ and $\varphi_{y}$, which are linear in the spin wave fields. The Néel vector in the rotated frame is, to the second order in the fluctuation,

$$
\tilde{\boldsymbol{n}}=\left(\begin{array}{c}
\varphi_{x} \\
\varphi_{y} \\
1-\varphi_{z}
\end{array}\right) .
$$

The longitudinal fluctuation $\varphi_{z}=\frac{1}{2}|\varphi|^{2}+O\left(\varphi^{3}\right)$ is of the second order, as required from the condition that $|\tilde{\boldsymbol{n}}|=1$, where $\boldsymbol{\varphi} \equiv$ $\left(\varphi_{x}, \varphi_{y}\right)$ is a two-component field of the spin wave. The representation (A3) is essentially the Holstein-Primakoff transformation. The local Néel vector in the laboratory frame $\boldsymbol{n}$ is written in terms of $\tilde{\boldsymbol{n}}$ in the rotated frame as

$$
\boldsymbol{n}=U \tilde{\boldsymbol{n}},
$$

where

$$
U=R_{z}\left(\phi_{0}\right) R_{y}\left(\theta_{0}\right)
$$


is a $3 \times 3$ rotation matrix connecting the direction $\left(\theta_{0}, \phi_{0}\right)$ to the $z$ axis.

$$
R_{i}(\theta)=e^{-i J_{i} \theta}
$$

is a rotation matrix around the $i$ axis by the angle $\theta$, where

$$
J_{x}=\left(\begin{array}{ccc}
0 & 0 & 0 \\
0 & 0 & -i \\
0 & i & 0
\end{array}\right), \quad J_{y}=\left(\begin{array}{ccc}
0 & 0 & i \\
0 & 0 & 0 \\
-i & 0 & 0
\end{array}\right), \quad J_{z}=\left(\begin{array}{ccc}
0 & -i & 0 \\
i & 0 & 0 \\
0 & 0 & 0
\end{array}\right)
$$

are generators of rotation satisfying

$$
\left[J_{i}, J_{j}\right]=i \epsilon_{i j k} J_{k}
$$

Explicitly,

$$
U(\theta, \phi)=\left(\begin{array}{ccc}
\cos \theta \cos \phi & -\sin \phi & \sin \theta \cos \phi \\
\cos \theta \sin \phi & \cos \phi & \sin \theta \sin \phi \\
-\sin \theta & 0 & \cos \theta
\end{array}\right) .
$$

As a result of the rotation, the derivative of the Néel vector becomes a covariant derivative with a gauge field [26] as

$$
\partial_{\mu} \boldsymbol{n}=U\left(\partial_{\mu}+i \mathcal{A}_{\mu}\right) \tilde{\boldsymbol{n}},
$$

where

$$
\begin{aligned}
\mathcal{A}_{\mu} & \equiv-i U^{-1} \partial_{\mu} U \\
& =-i\left(\begin{array}{ccc}
0 & -\cos \theta_{0} \partial_{\mu} \phi_{0} & \partial_{\mu} \theta_{0} \\
\cos \theta_{0} \partial_{\mu} \phi_{0} & 0 & \sin \theta_{0} \partial_{\mu} \phi_{0} \\
-\partial_{\mu} \theta_{0} & -\sin \theta_{0} \partial_{\mu} \phi_{0} & 0
\end{array}\right)
\end{aligned}
$$

is the gauge field in the $3 \times 3$ matrix representation. The derivative term of the Lagrangian is

$$
\left(\partial_{\mu} \boldsymbol{n}\right)^{2}=\left(\partial_{\mu} \tilde{\boldsymbol{n}}\right)^{2}-i\left[\tilde{\boldsymbol{n}} \mathcal{A}_{\mu} \partial_{\mu} \tilde{\boldsymbol{n}}-\left(\tilde{\boldsymbol{n}} \overleftarrow{\partial_{\mu}}\right) \mathcal{A}_{\mu} \tilde{\boldsymbol{n}}\right]+\tilde{\boldsymbol{n}}\left(\mathcal{A}_{\mu}\right)^{2} \tilde{\boldsymbol{n}}
$$

\section{Magnon expansion}

Here we include for generality a hard-axis anisotropy energy along the $y$ direction, described by a Hamiltonian

$$
H_{K_{\perp}}=\frac{J}{2 a} \int d x \frac{\kappa}{\lambda_{0}^{2}}\left(n_{y}\right)^{2},
$$

where the hard-axis energy is $J \kappa / \lambda_{0}^{2}$. The total Lagrangian in terms of domain wall coordinates $\left(\theta_{0}, \phi_{0}\right)$ and spin wave variable $\varphi$ reads

$$
\begin{aligned}
L= & L^{(0)}+L^{(1)}+L^{(2)}, \\
L^{(0)} \equiv & \frac{J}{2 a} \int d x\left\{\frac{1}{c^{2}}\left(\dot{\theta}_{0}^{2}+\sin ^{2} \theta_{0} \dot{\phi}_{0}^{2}\right)-\left[\left(\nabla \theta_{0}\right)^{2}+\sin ^{2} \theta_{0}\left(\nabla \phi_{0}\right)^{2}\right]-\frac{1}{\lambda_{0}^{2}} \sin ^{2} \theta_{0}\left(1+\kappa \sin ^{2} \phi_{0}\right)\right\}, \\
L^{(1)} \equiv & \frac{J}{2 a} \int d x\left\{2\left[\frac{1}{c^{2}}\left(\dot{\theta}_{0} \dot{\varphi}_{x}+\sin \theta_{0} \dot{\phi}_{0} \dot{\varphi}_{y}\right)-\left(\nabla \theta_{0} \nabla \varphi_{x}+\sin \theta_{0} \nabla \phi_{0} \nabla \varphi_{y}\right)\right]+\frac{1}{c^{2}}\left[\sin 2 \theta_{0}\left(\dot{\phi}_{0}\right)^{2} \varphi_{x}-2 \cos \theta_{0} \dot{\theta_{0}} \dot{\phi}_{0} \varphi_{y}\right]\right. \\
& \left.-\left[\sin 2 \theta_{0}\left(\nabla \phi_{0}\right)^{2} \varphi_{x}-2 \cos \theta_{0} \nabla \theta_{0} \nabla \phi_{0} \varphi_{y}\right]-\frac{1}{\lambda_{0}^{2}}\left[\sin 2 \theta_{0} \varphi_{x}\left(1+\kappa \sin ^{2} \phi_{0}\right)+\kappa \sin \theta_{0} \sin 2 \phi_{0} \varphi_{y}\right]\right\}, \\
L^{(2)}= & \frac{J}{2 a} \int d x\left(\frac{1}{c^{2}}|\dot{\varphi}|^{2}-|\nabla \varphi|^{2}+2 \cos \theta_{0}\left[\frac{1}{c^{2}} \dot{\phi_{0}}\left(\varphi_{x} \stackrel{\leftrightarrow}{\partial_{t}} \varphi_{y}\right)-\nabla \phi_{0}\left(\varphi_{x} \stackrel{\leftrightarrow}{\nabla} \varphi_{y}\right)\right]\right. \\
& +\varphi_{x}^{2} \cos 2 \theta_{0}\left\{\left[\frac{\dot{\phi}_{0}{ }^{2}}{c^{2}}-\left(\nabla \phi_{0}\right)^{2}\right]-\frac{1}{\lambda_{0}^{2}}\left(1+\kappa \sin ^{2} \phi_{0}\right)\right\}+\varphi_{y}^{2}\left\{-\left[\frac{\dot{\theta_{0}}}{c^{2}}-\left(\nabla \theta_{0}\right)^{2}\right]+\cos ^{2} \theta_{0}\left[\frac{\dot{\phi}_{0}{ }^{2}}{c^{2}}-\left(\nabla \phi_{0}\right)^{2}\right]\right. \\
& \left.\left.-\frac{1}{\lambda_{0}^{2}}\left[\cos ^{2} \theta_{0}+\kappa\left(\cos ^{2} \phi_{0}-\sin ^{2} \theta_{0} \sin ^{2} \phi_{0}\right)\right]\right\}+\varphi_{x} \varphi_{y}\left\{2 \sin \theta_{0}\left[\frac{1}{c^{2}} \dot{\theta_{0}} \dot{\phi}_{0}-\left(\nabla \theta_{0}\right)\left(\nabla \phi_{0}\right)\right]-\frac{1}{\lambda_{0}^{2}} \kappa \cos \theta_{0} \sin 2 \phi_{0}\right\}\right) .
\end{aligned}
$$

The second-order contributions containing $\left(\varphi_{x} \stackrel{\leftrightarrow}{\partial_{\mu}} \varphi_{y}\right)$ are the effect of magnon current (or density if $\mu=t$ ) inducing a torque for the Néel order parameter (spin-transfer effect of spin waves). The coupling between the two spin wave modes $\varphi_{x}$ and $\varphi_{y}$ describes 
the process of angular momentum transfer, as they carry opposite angular momenta [12]. The $\left(\varphi_{x} \overleftrightarrow{\partial_{\mu}} \varphi_{y}\right)$ term indicates that the spin Berry phase $\cos \theta_{0} \partial_{\mu} \phi_{0}$ acts as an effective magnetic field, inducing a coupling $\varphi_{x} \partial_{\mu} \varphi_{y}$.

\section{Linear coupling}

The equations of motion for the classical variables derived from $L^{(0)}$ are

$$
\begin{array}{r}
-2\left(\frac{1}{c^{2}} \ddot{\theta_{0}}-\nabla^{2} \theta_{0}\right)+\sin 2 \theta_{0}\left[\frac{1}{c^{2}}\left(\dot{\phi}_{0}\right)^{2}-\left(\nabla \phi_{0}\right)^{2}\right]-\frac{1}{\lambda_{0}^{2}} \sin 2 \theta_{0}\left(1+\kappa \sin ^{2} \phi_{0}\right)=0, \\
-2\left[\frac{1}{c^{2}} \partial_{t}\left(\sin ^{2} \theta_{0} \dot{\phi}_{0}\right)-\nabla\left(\sin ^{2} \theta_{0} \nabla \phi_{0}\right)\right]-\frac{1}{\lambda_{0}^{2}} \kappa \sin ^{2} \theta_{0} \sin 2 \phi_{0}=0 .
\end{array}
$$

The linear contribution is written using integral by parts in the action (time integral of the Lagrangian) as

$$
\begin{aligned}
\int d t L^{(1)}= & \frac{J}{2 a} \int d t \int d x\left(\varphi_{x}\left\{-2\left(\frac{1}{c^{2}} \ddot{\theta}_{0}-\nabla^{2} \theta_{0}\right)+\sin 2 \theta_{0}\left[\frac{1}{c^{2}}\left(\dot{\phi}_{0}\right)^{2}-\left(\nabla \phi_{0}\right)^{2}\right]-\frac{1}{\lambda_{0}^{2}} \sin 2 \theta_{0}\left(1+\kappa \sin ^{2} \phi_{0}\right)\right\}\right. \\
& \left.+\varphi_{y}\left\{\frac{2}{\sin \theta_{0}}\left[-\frac{1}{c^{2}} \partial_{t}\left(\sin ^{2} \theta_{0} \dot{\phi}_{0}\right)+\nabla\left(\sin ^{2} \theta_{0} \nabla \phi_{0}\right)\right]-\frac{1}{\lambda_{0}^{2}} \kappa \sin \theta_{0} \sin 2 \phi_{0}\right\}\right) .
\end{aligned}
$$

Using Eq. (A15), the linear coupling term (A16) vanishes if $\theta_{0}$ and $\phi_{0}$ are a classical solution; $L^{(1)}=0$. The absence of the linear term for a classical solution is natural mathematically. This fact does not necessary prohibit magnon emission from the wall, as the solution of the equation of motion may contain an extended state corresponding to the propagating magnons. (Emission arises also from higher-order interactions.) In fact, the soliton solution, which is strictly localized $\left[\propto e^{-|x| / \ell}\right.$ at $|x| \rightarrow \infty(\ell$ is a constant)], exists only under particular conditions, and the solution of the accelerating domain wall may in general have an extended solution with magnons. This is supported by the following simple argument. Consider the case of the sine-Gordon model, corresponding to that of angle $\theta$ with $\phi=0$ :

$$
\frac{\ddot{\theta}}{c^{2}}-\nabla^{2} \theta+\frac{1}{\lambda_{0}^{2}} \sin \theta \cos \theta=0 .
$$

We are interested in the behavior at $|x| \rightarrow \infty$, where $\sin \theta \rightarrow 0$. Choosing $\theta \simeq 0$ therefore, the equation has a wave solution $e^{i(k x-\omega t)}$ with finite real parts of $k$ and $\omega$ if

$$
\frac{\omega}{c}= \pm \sqrt{k^{2}+\left(\lambda_{0}\right)^{-2}}
$$

If an acceleration angular frequency is larger than the magnon gap, $c / \lambda_{0}$, therefore, the equation has an extended solution. In contrast, the static solution has $k= \pm i / \lambda_{0}$, i.e., only localized structure. Therefore, the dynamic solution may contain magnons besides a localized structure, and this solution corresponds to magnon emission. Usually, to access such emission solution analytically is difficult. The collective coordinate formulation provides a practical approximation scheme to treat the emission, by separating the localized mode and fluctuation. The solution represented by collective coordinates is not a rigorous solution, and thus linear coupling to the spin wave appears. Nevertheless, the calculation in this approach is straightforward and useful. The coupling depends on the choice of the collective coordinates, and this would be the reason why there are theories discussing different couplings [6-8].

\section{Equations for DW collective coordinates}

Based on these considerations, we apply collective coordinate representation of a domain wall for $\left(\theta_{0}, \phi_{0}\right)$, representing the total Néel vector as the sum of the domain wall configuration and spin wave $\varphi$. The domain wall solution is

$$
\cos \theta_{0}=\tanh \frac{x-X(t)}{\lambda(t)}, \quad \sin \theta_{0}=\frac{1}{\cosh \frac{x-X(t)}{\lambda(t)}}, \quad \phi_{0}=\phi_{0}(t) .
$$

We let the thickness $\lambda$ be dynamic besides $X$ and $\phi_{0}$ to include deformation effects [8]. We then have

$$
\begin{aligned}
\nabla \theta_{0} & =-\frac{1}{\lambda} \frac{1}{\cosh u}, \quad \nabla^{2} \theta_{0}=\frac{1}{\lambda^{2}} \frac{\sinh u}{\cosh ^{2} u}, \\
\dot{\theta_{0}} & =\frac{1}{\lambda}(\dot{X}+u \dot{\lambda}) \frac{1}{\cosh u}, \\
\ddot{\theta_{0}} & =\frac{1}{\lambda}\left[\ddot{X}+u \ddot{\lambda}-2 \frac{\dot{\lambda}}{\lambda}(\dot{X}+u \dot{\lambda})\right] \frac{1}{\cosh u}+\frac{1}{\lambda^{2}}(\dot{X}+u \dot{\lambda})^{2} \frac{\sinh u}{\cosh ^{2} u},
\end{aligned}
$$

where $u \equiv \frac{x-X(t)}{\lambda(t)}$. 
The domain wall part of the Lagrangian is

$$
L^{(0)}=\frac{M_{\mathrm{w}}}{2} \dot{X}^{2}-\frac{J}{a \lambda}\left[1-\left(\frac{\lambda}{\lambda_{0}}\right)^{2}\right],
$$

where $M_{\mathrm{w}} \equiv \frac{2 J}{a c^{2} \lambda}=\frac{2 \hbar^{2}}{J_{0} \lambda a}$ is the wall mass. As $\lambda \rightarrow 0$, i.e., as the wall speed $V_{\mathrm{w}} \rightarrow c$, the wall mass diverges, consistent with the relativistic form of the Lagrangian [3]. The contribution of $\dot{X}$ of the dissipation function is

$$
W=\frac{\alpha}{\lambda a} \dot{X}^{2},
$$

and the equation of motion for $X$ determined by $L^{(0)}$ and $W$ is

$$
M_{\mathrm{w}} \ddot{X}=-\alpha_{\mathrm{w}} \dot{X}
$$

where $\alpha_{\mathrm{w}}=\frac{2 \alpha}{\lambda a}$.

For a domain wall solution with $\phi_{0}=0$, the second-order part describing the magnon is, neglecting explicit couplings to $\dot{X}$ and $\dot{\lambda}$,

$$
L^{(2)}=\frac{J}{2 a} \int d x\left\{\frac{1}{c^{2}}|\dot{\varphi}|^{2}-|\nabla \varphi|^{2}-\frac{1}{\lambda_{0}^{2}}\left[\varphi_{x}^{2}\left(1-\frac{2}{\cosh ^{2} u}\right)+\varphi_{y}^{2}\left[1+\kappa-\frac{1}{\cosh ^{2} u}\left(1+\frac{\lambda_{0}^{2}}{\lambda^{2}}\right)\right]\right]\right\} .
$$

The magnon dispersion is therefore determined by

$$
L_{\varphi}=\frac{1}{2 g} \sum_{i, k}\left\{\left[\dot{\varphi}_{i}(k)\right]^{2}-\left(\omega_{k}^{(i)}\right)^{2}\left[\varphi_{i}(k)\right]^{2}\right\},
$$

where $i=x, y, g \equiv c^{2} / J$, and

$$
\omega_{k}^{(x)} \equiv \sqrt{c^{2}\left[k^{2}+\left(\lambda_{0}\right)^{-2}\right]}, \quad \omega_{k}^{(y)} \equiv \sqrt{c^{2}\left[k^{2}+\left(\lambda_{0}\right)^{-2}(1+\kappa)\right]}
$$

are magnon energies in the $x$ and $y$ directions, respectively. The remaining term of Eq. (A24) represents the domain wall potential term (approximating $\lambda=\lambda_{0}$ ),

$$
V=-K \int \frac{d x}{a} \frac{1}{\cosh ^{2} u}\left(\varphi_{x}^{2}+\varphi_{y}^{2}\right)
$$

which is discussed in detail in the main text $\left(K=J / \lambda_{0}^{2}\right)$.

The linear term of the Lagrangian, Eq. (A16), reads in terms of domain wall coordinates

$$
\begin{aligned}
L^{(1)}=\frac{J}{a} \int d x & \varphi_{x}\left\{-\frac{u}{\cosh u} \frac{1}{c^{2} \lambda}\left(\ddot{\lambda}-2 \frac{\dot{\lambda}^{2}}{\lambda}\right)+\frac{\tanh u}{\cosh u}\left[-\frac{1}{c^{2} \lambda^{2}}(\dot{X}+u \dot{\lambda})^{2}+\frac{1}{\lambda^{2}}-\frac{1}{\lambda_{0}^{2}}\left(1+\kappa \sin ^{2} \phi_{0}\right)+\frac{1}{c^{2}}\left(\dot{\phi}_{0}\right)^{2}\right]\right\} \\
& \left.-\varphi_{y} \frac{\tanh u}{\cosh u} \frac{2}{c^{2} \lambda} \dot{\phi}_{0}(\dot{X}+u \dot{\lambda})\right),
\end{aligned}
$$

where we used the orthogonality between the $\varphi_{i}$ and the zero mode, $1 / \cosh u, \int d u \frac{\varphi_{i}}{\cosh u}=0$. As seen, $\varphi_{x}$ couples to dynamics of $X$ and $\lambda$, while $\varphi_{y}$ couples to that of $\phi_{0}$.

The equations of motion with dissipation included for the domain wall configuration are

$$
\begin{gathered}
\frac{1}{\cosh u}\left(\frac{\left[\ddot{X}+u \ddot{\lambda}-2 \frac{\dot{\lambda}}{\lambda}(\dot{X}+u \dot{\lambda})\right]}{c^{2} \lambda}+\frac{\alpha}{J} \frac{\dot{X}+u \dot{\lambda}}{\lambda}\right)+\frac{\tanh u}{\cosh u}\left[-\frac{(\dot{X}+u \dot{\lambda})^{2}}{c^{2} \lambda^{2}}+\frac{1}{\lambda^{2}}-\frac{1}{\lambda_{0}^{2}}\left(1+\kappa \sin ^{2} \phi_{0}\right)+\frac{\left(\dot{\phi}_{0}\right)^{2}}{c^{2}}\right]=0 \\
\frac{1}{\cosh u}\left(\frac{\ddot{\phi_{0}}}{c^{2}}+\frac{\kappa}{2 \lambda_{0}^{2}} \sin 2 \phi_{0}+\frac{1}{J} \alpha \dot{\phi_{0}}\right)+\frac{\tanh u}{\cosh u} \frac{2}{c^{2} \lambda} \dot{\phi}_{0}(\dot{X}+u \dot{\lambda})=0 .
\end{gathered}
$$

Obviously, this equation cannot generally be satisfied locally for each position $x$ by any choice of $X, \lambda$, and $\phi_{0}$. What we can impose in terms of the collective coordinates are the averaged condition. Integrating over $x$, these equations reduce to

$$
\begin{aligned}
\ddot{X}-\frac{\dot{\lambda}}{\lambda} \dot{X}+\frac{\alpha}{J} \dot{X} & =0, \\
\frac{\ddot{\phi_{0}}}{c^{2}}+\frac{\kappa}{2 \lambda_{0}^{2}} \sin 2 \phi_{0}+\frac{1}{J} \alpha \dot{\phi}_{0}+2 \frac{\dot{\phi}_{0} \dot{\lambda}}{c^{2} \lambda} & =0,
\end{aligned}
$$

where we used $\frac{1}{\lambda} \int d x u \frac{\tanh u}{\cosh u}=\pi$. The first equation is a sort of trivial one without a driving force. We see from Eq. (A31) that the wall dynamics in antiferromagnets is qualitatively different from the one in ferromagnets; the position $X$ and the angle $\phi_{0}$ 
are decoupled for low wall velocity, while they are canonically conjugate to each other in the ferromagnetic case. Picking up the asymmetric part of Eq. (A30) by multiplying by $\tanh u$ and integrating over $x$, we obtain

$$
\begin{aligned}
\frac{2}{c^{2}} \lambda \ddot{\lambda}+\frac{2 \alpha}{J} \lambda \dot{\lambda}+\frac{1}{c^{2}} \mu_{\lambda} \dot{\lambda}^{2}+\frac{\lambda^{2}}{\lambda_{0}^{2}}\left(1+\kappa \sin ^{2} \phi_{0}\right)-\left(1-\frac{\dot{X}^{2}}{c^{2}}\right)-\frac{\lambda^{2}}{c^{2}}\left(\dot{\phi}_{0}\right)^{2}=0, \\
\dot{\phi_{0} \dot{X}}=0,
\end{aligned}
$$

where $\mu_{\lambda}=\pi\left(1+\frac{\pi^{2}}{8}\right)-4$. We used

$$
\frac{1}{\lambda} \int d x \frac{\tanh ^{2} u}{\cosh u}=\frac{\pi}{2}, \quad \frac{1}{\lambda} \int d x u^{2} \frac{\tanh ^{2} u}{\cosh u}=\pi\left(1+\frac{\pi^{2}}{8}\right) .
$$

The last equation of Eq. (A32) leads to a decoupling of $\phi_{0}$ from $X$ and $\lambda$; in fact, choosing $\dot{\phi}_{0}=0$ leads to $\phi_{0}=0$ resulting in

$$
\begin{aligned}
\frac{2}{c^{2}} \lambda \ddot{\lambda}+\frac{2 \alpha}{J} \lambda \dot{\lambda}+\frac{1}{c^{2}} \mu_{\lambda} \dot{\lambda}^{2}+\frac{\lambda^{2}}{\lambda_{0}^{2}}-\left(1-\frac{\dot{X}^{2}}{c^{2}}\right) & =0, \\
\ddot{X}-\frac{\dot{\lambda}}{\lambda} \dot{X}+\frac{\alpha}{J} \dot{X} & =0 .
\end{aligned}
$$

Using these equations, the linear coupling of Eq. (A28) reads

$$
L^{(1)}=\frac{J}{a} \frac{1}{c^{2}} \int d x \varphi_{x}\left\{-\frac{u}{\cosh u}\left(\frac{\ddot{\lambda}}{\lambda}-2 \frac{\dot{\lambda}^{2}}{\lambda^{2}}\right)+\frac{\tanh u}{\cosh u}\left[2 \frac{\ddot{\lambda}}{\lambda}+\left(\mu_{\lambda}-u^{2}\right) \frac{\dot{\lambda}^{2}}{\lambda^{2}}-2 u \frac{\dot{X} \dot{\lambda}}{\lambda^{2}}\right]\right\}
$$

with vanishing $\varphi_{y}$ term. As we see here, the linear couplings are with second-order time derivatives like $\ddot{\lambda}$ and $\dot{\lambda}^{2}$. This is a result of the relativistic form of the magnon Lagrangian in antiferromagnets, and this leads to non-Ohmic weak dissipation as shown in Appendix B. In the ferromagnetic case, in contrast, the coupling is to first-order time derivatives like $\dot{\lambda}$, resulting in an Ohmic dissipation and a Gilbert damping constant. The last term of Eq. (A35) is a coupling between $\varphi_{x}$ and $\ddot{X}$ if we use the equation of motion, Eq. (A34). It is seen that $\ddot{X}$ induces a magnon mode $\varphi_{x}$ with a spatial profile symmetric with respect to the wall center, while $\ddot{\lambda}$ induces an asymmetric mode. As seen from this result, variation of $\lambda$ is essential for magnon emission. This is the same as in the ferromagnetic case [8]. In the present antiferromagnetic case, this is natural also from the Lorentz invariance, constant velocity motion is a stable solution, and no emission is allowed. As we have argued, this linear-coupling Lagrangian is a model derived for our choice of the collective coordinates, and may not be something universal.

\section{APPENDIX B: EMISSION DUE TO LINEAR COUPLING}

Let us study the spin wave emission from the linear coupling, Eq. (A35). The calculation is parallel to the ferromagnetic case studied in Ref. [8]. Here we focus on the effect of center of mass motion, as the $\lambda$ dynamics is an internal degree of freedom not directly detectable. Using the last equation of Eq. (A34) and neglecting the terms that do not depend on $X$ in Eq. (A35), we see that acceleration of the wall leads to a linear coupling of

$$
L_{X}^{(1)}=-\frac{2 J}{a} \frac{1}{c^{2}}\left(\int d x u \frac{\tanh u}{\cosh u} \varphi_{x}\right) \frac{\dot{X} \dot{\lambda}}{\lambda^{2}}=f_{X}\left(\int d u u \frac{\tanh u}{\cosh u} \varphi_{x}\right) \ddot{X},
$$

where $f_{X} \equiv-\frac{2 J}{a} \frac{1}{c^{2}}$. As this expression indicates, the acceleration induces a spatially symmetric spin wave profile. The force due to emission is

$$
F_{X}=\frac{\partial}{\partial X} L_{X}^{(1)}=f_{X} \frac{\partial^{2}}{\partial t^{2}} \bar{\varphi}_{X}
$$

where

$$
\bar{\varphi}_{X} \equiv \int d u u \frac{\tanh u}{\cosh u}\left\langle\varphi_{x}\right\rangle,
$$

being a spin wave expectation value $\left\langle\varphi_{x}\right\rangle$ with a spatially symmetric weight around the wall. Here we used the integration by parts with respect to time considering the action (the time integral of the Lagrangian). The expectation value $\left\langle\varphi_{x}\right\rangle$ is expressed in terms of the retarded Green's function of spin wave mode $\varphi_{x}$,

$$
G_{\varphi_{x}}^{\mathrm{r}}\left(u, t, u^{\prime}, t^{\prime}\right) \equiv-i \theta\left(t-t^{\prime}\right)\left\langle\left[\varphi_{x}(u, t), \bar{\varphi}_{x}\left(u^{\prime}, t^{\prime}\right)\right]\right\rangle,
$$

as [8]

$$
\left\langle\varphi_{x}(x, t)\right\rangle=f_{X} \int_{-\infty}^{t} d t^{\prime} \ddot{X}\left(t^{\prime}\right) \int \frac{d x^{\prime}}{\lambda\left(t^{\prime}\right)} \frac{x^{\prime}-X\left(t^{\prime}\right)}{\lambda\left(t^{\prime}\right)} \frac{\tanh \frac{x^{\prime}-X\left(t^{\prime}\right)}{\lambda\left(t^{\prime}\right)}}{\cosh \frac{x^{\prime}-X\left(t^{\prime}\right)}{\lambda\left(t^{\prime}\right)}} G_{\varphi_{x}}^{\mathrm{r}}\left(x, t, x^{\prime}, t^{\prime}\right) .
$$


This expression indicates that the wall acceleration at time $t^{\prime}<t$ emits spin waves around the wall center $X\left(t^{\prime}\right)$ and the spin waves propagate to the point of the observation $x$ at time $t$.

Although there is an analytical solution of the spin wave in a $\cosh ^{-1}$ potential [Eq. (A24) with $\dot{X}=\dot{\lambda}=0$ ] [8,20], we here expand the spin waves by plane waves. In fact, description in terms of the correct wave function depends on the wall position $X$ at each time, and thus is not convenient to represent emitted waves. The retarded Green's function in the angular frequency representation is thus

$$
G_{\varphi_{x}}^{\mathrm{r}}\left(x, x^{\prime}, \omega\right)=2 c^{2} \sum_{k} \frac{1}{(\omega+i 0)^{2}-\left(\omega_{k}^{(x)}\right)^{2}} e^{i k\left(x-x^{\prime}\right)} e^{-i \omega\left(t-t^{\prime}\right)},
$$

where $\omega_{k}^{(x)}=\sqrt{c^{2}\left[k^{2}+\left(\lambda_{0}\right)^{-2}\right]}$ is the spin wave energy of the mode in the $x$ direction and thus

$$
\left\langle\varphi_{x}(x, t)\right\rangle=2 c^{2} f_{X} \int_{-\infty}^{t} d t^{\prime} \ddot{X}\left(t^{\prime}\right) \int \frac{d \omega}{2 \pi} \sum_{k} \Phi_{k}\left(t^{\prime}\right) \frac{e^{-i \omega\left(t-t^{\prime}\right)}}{(\omega+i 0)^{2}-\left(\omega_{k}^{(x)}\right)^{2}} e^{i k\left[x-X\left(t^{\prime}\right)\right]}
$$

and

$$
\Phi_{k}\left(t^{\prime}\right)=\int d u^{\prime} u^{\prime} \frac{\tanh u^{\prime}}{\cosh u^{\prime}} e^{-i k u^{\prime} \lambda\left(t^{\prime}\right)}=\frac{\pi}{\cosh \frac{\pi}{2} k \lambda\left(t^{\prime}\right)}\left[1-\frac{\pi}{2} k \lambda\left(t^{\prime}\right) \tanh \frac{\pi}{2} k \lambda\left(t^{\prime}\right)\right] ;
$$

$u^{\prime} \equiv \frac{x^{\prime}-X\left(t^{\prime}\right)}{\lambda\left(t^{\prime}\right)}$ is a form factor representing the distribution of $k$ emitted. After the integration over $\omega$, we have

$$
\left\langle\varphi_{x}(x, t)\right\rangle=2 i c^{2} f_{X} \int_{-\infty}^{t} d t^{\prime} \ddot{X}\left(t^{\prime}\right) \sum_{k} \frac{\Phi_{k}\left(t^{\prime}\right)}{\omega_{k}^{(x)}}\left(e^{-i \omega_{k}^{(x)}\left(t-t^{\prime}\right)}-e^{i \omega_{k}^{(x)}\left(t-t^{\prime}\right)}\right) e^{i k\left[x-X\left(t^{\prime}\right)\right]},
$$

as the amplitude of the linearly created magnon.

\section{Dissipation in the instantaneous approximation}

In the instantaneous approximation corresponding to slow excitations, the retardation effect in $G^{\mathrm{r}}$ is neglected [8], resulting in

$$
\left\langle\varphi_{x}(x, t)\right\rangle=-2 c^{2} f_{X} \ddot{X}(t) \sum_{k} \frac{\Phi_{k}(t)}{\left(\omega_{k}^{(x)}\right)^{2}} e^{i k[x-X(t)]} .
$$

The averaged density $\bar{\varphi}_{X}$ then reads

$$
\bar{\varphi}_{X}=-f_{X} \ddot{X}(t) \Phi_{X},
$$

where $\Phi_{X} \equiv 2 c^{2} \sum_{k} \frac{\left|\Phi_{k}(t)\right|^{2}}{\left(\omega_{k}^{(x)}\right)^{2}}$. The reaction force due to emission, Eq. (A35), is [assuming that the time dependence of $\Phi_{k}(t)$ is weak]

$$
F_{X}=-f_{X} \Phi_{X} \frac{\partial^{4} X}{\partial t^{4}}
$$

It is an even higher-order derivative than the force proportional to $\frac{\partial^{3} X}{\partial t^{3}}$ found in the ferromagnetic case [7], and is weak for low-frequency dynamics. The energy dissipation rate, $\frac{d \mathcal{E}}{d t}=F_{X} \dot{X}$, due to this linear emission is proportional (after integral by parts) to $\frac{d}{d t}\left(\ddot{X}^{2}\right)$. The dissipation arising from the acceleration is therefore negligibly small at low energy compared to the Ohmic damping proportional to $\frac{d}{d t}\left(\dot{X}^{2}\right)$. This is in contrast to the ferromagnetic case, where Ohmic damping is present [8].

\section{APPENDIX C: EVALUATION OF MAGNON AMPLITUDES}

The scattering amplitude (or the induced spin wave density) by the moving wall is

$$
\left\langle a_{k+q}^{(i) \dagger} a_{k}^{(i)}\right\rangle=i G_{k, k+q}^{(i)<}(t, t),
$$

where $G_{k, k+q}^{(i)<}\left(t, t^{\prime}\right) \equiv-i\left\langle a_{k+q}^{(i) \dagger}\left(t^{\prime}\right) a_{k}^{(i)}(t)\right\rangle$ is the lesser Green's function. The total wave vector of the spin wave increases by $q$ as a result of interaction, and thus $\hbar q$ is the momentum given to the spin waves from the wall. In the linear response to the potential, it is

$$
\begin{aligned}
\left\langle a_{k+q}^{(i) \dagger} a_{k}^{(i)}\right\rangle(t) & =i \frac{K g}{a} \int_{C} d t^{\prime}\left\langle a_{k+q}^{(i) \dagger}(t) a_{k}^{(i)}(t) a_{k+q}^{(i)}\left(t^{\prime}\right) a_{k}^{(i) \dagger}\left(t^{\prime}\right)\right| \lambda\left(t^{\prime}\right) \frac{W_{-q} e^{i q X\left(t^{\prime}\right)}}{\sqrt{\omega_{k}^{(i)} \omega_{k+q}^{(i)}}} \\
& =-i \frac{K g}{a} \int_{C} d t^{\prime} \lambda\left(t^{\prime}\right) \frac{W_{-q} e^{i q X\left(t^{\prime}\right)}}{\sqrt{\omega_{k}^{(i)} \omega_{k+q}^{(i)}}}\left[g_{k}\left(t, t^{\prime}\right) g_{k+q}\left(t^{\prime}, t\right)\right]^{<}
\end{aligned}
$$


where $g_{k}\left(t, t^{\prime}\right)$ denotes the free-path-ordered magnon Green's function defined on path $C$ in a plane of a complex time. The lesser component is calculated using $\int_{C} d t^{\prime}\left[g_{k}\left(t, t^{\prime}\right) g_{k+q}\left(t^{\prime}, t\right)\right]^{<}=\int_{-\infty}^{\infty} d t^{\prime}\left[g_{k}^{\mathrm{r}}\left(t, t^{\prime}\right) g_{k+q}^{<}\left(t^{\prime}, t\right)+g_{k}^{<}\left(t, t^{\prime}\right) g_{k+q}^{\mathrm{a}}\left(t^{\prime}, t\right)\right]$, and Green's functions on real time are

$$
g_{\boldsymbol{k} \omega}^{\mathrm{r}}=\frac{1}{\omega-\omega_{k}+i \eta}, \quad g_{\boldsymbol{k} \omega}^{<}=-n\left(\omega_{k}\right)\left(g_{\boldsymbol{k} \omega}^{\mathrm{a}}-g_{\boldsymbol{k} \omega}^{\mathrm{r}}\right),
$$

where $n(\omega) \equiv\left[e^{\beta \omega}-1\right]^{-1}$ is the Bose distribution function, $g_{k \omega}^{\mathrm{r}}$ and $g_{\boldsymbol{k} \omega}^{\mathrm{a}}$ are the retarded and advanced Green's functions, respectively, and $\eta$ is a damping parameter. We therefore obtain

$$
\begin{aligned}
\left\langle a_{k+q}^{(i) \dagger} a_{k}^{(i)}\right\rangle(t) & =i \frac{K g}{a} \int_{-\infty}^{\infty} d t^{\prime} \lambda\left(t^{\prime}\right) \frac{W_{-q} e^{i q X\left(t^{\prime}\right)}}{\sqrt{\omega_{k}^{(i)} \omega_{k+q}^{(i)}}} \int \frac{d \Omega}{2 \pi} \int \frac{d \omega}{2 \pi} e^{i \Omega\left(t-t^{\prime}\right)}\left(n_{k+q}-n_{k}\right) g_{k \omega}^{\mathrm{r}} g_{k+q, \omega+\Omega}^{\mathrm{a}} \\
& =-\frac{K g}{a} \int_{-\infty}^{\infty} d t^{\prime} \lambda\left(t^{\prime}\right) \frac{W_{-q} e^{i q X\left(t^{\prime}\right)}}{\sqrt{\omega_{k}^{(i)} \omega_{k+q}^{(i)}}} \int \frac{d \Omega}{2 \pi} e^{i \Omega\left(t-t^{\prime}\right)} \frac{n_{k+q}-n_{k}}{\omega_{k+q}-\omega_{k}-\Omega+2 i \eta} .
\end{aligned}
$$

Similarly the amplitudes of the two-magnon annihilation and creation are

$$
\left\langle a_{-(k+q)}^{(i)} a_{k}^{(i)}\right\rangle(t)=i \frac{K g}{a} \int_{C} d t^{\prime}\left\langle a_{-(k+q)}^{(i)}(t) a_{k}^{(i)}(t) a_{-(k+q)}^{(i) \dagger}\left(t^{\prime}\right) a_{k}^{(i) \dagger}\left(t^{\prime}\right)\right| \lambda\left(t^{\prime}\right) \frac{W_{-q} e^{i q X\left(t^{\prime}\right)}}{\sqrt{\omega_{k}^{(i)} \omega_{k+q}^{(i)}}}
$$

and

$$
\left\langle a_{(k+q)}^{(i) \dagger} a_{-k}^{(i) \dagger}\right\rangle(t)=i \frac{K g}{a} \int_{C} d t^{\prime}\left\langle a_{(k+q)}^{(i) \dagger}(t) a_{-k}^{(i) \dagger}(t) a_{(k+q)}^{(i)}\left(t^{\prime}\right) a_{-k}^{(i)}\left(t^{\prime}\right)\right| \lambda\left(t^{\prime}\right) \frac{W_{-q} e^{i q X\left(t^{\prime}\right)}}{\sqrt{\omega_{k}^{(i)} \omega_{k+q}^{(i)}}},
$$

respectively. The expectation value is decomposed using

$$
\begin{aligned}
& \theta\left(t-t^{\prime}\right)\left[g^{>}\left(t, t^{\prime}\right)-g^{<}\left(t, t^{\prime}\right)\right]=g^{\mathrm{r}}\left(t, t^{\prime}\right), \\
& \theta\left(t-t^{\prime}\right)\left[g^{>}\left(t^{\prime}, t\right)-g^{<}\left(t^{\prime}, t\right)\right]=-g^{\mathrm{a}}\left(t^{\prime}, t\right),
\end{aligned}
$$

as

$$
\begin{aligned}
\int_{C} d t^{\prime}\left\langle a_{-(k+q)}^{(i)}(t) a_{k}^{(i)}(t) a_{-(k+q)}^{(i) \dagger}\left(t^{\prime}\right) a_{k}^{(i) \dagger}\left(t^{\prime}\right)\right\rangle= & \int_{-\infty}^{t} d t^{\prime}\left\langle a_{k}^{(i)}(t) a_{k}^{(i) \dagger}\left(t^{\prime}\right)\right\rangle\left\langle a_{-(k+q)}^{(i)}(t) a_{-(k+q)}^{(i) \dagger}\left(t^{\prime}\right)\right\rangle \\
& +\int_{t}^{-\infty} d t^{\prime}\left\langle a_{k}^{(i) \dagger}\left(t^{\prime}\right) a_{k}^{(i)}(t)\right\rangle\left\langle a_{-(k+q)}^{(i) \dagger}\left(t^{\prime}\right) a_{-(k+q)}^{(i)}(t)\right\rangle \\
= & -\int_{-\infty}^{\infty} d t^{\prime}\left[g_{k}^{(i) \mathrm{r}}\left(t, t^{\prime}\right) g_{-(k+q)}^{(i)>}\left(t, t^{\prime}\right)+g_{k}^{(i)<}\left(t, t^{\prime}\right) g_{-(k+q)}^{(i) \mathrm{r}}\left(t, t^{\prime}\right)\right], \\
\int_{C} d t^{\prime}\left\langle a_{(k+q)}^{(i) \dagger}(t) a_{-k}^{(i) \dagger}(t) a_{(k+q)}^{(i)}\left(t^{\prime}\right) a_{-k}^{(i)}\left(t^{\prime}\right)\right\rangle= & \int_{-\infty}^{t} d t^{\prime}\left\langle a_{-k}^{(i) \dagger}(t) a_{-k}^{(i)}\left(t^{\prime}\right)\right\rangle\left\langle a_{(k+q)}^{(i) \dagger}(t) a_{(k+q)}^{(i)}\left(t^{\prime}\right)\right\rangle \\
& +\int_{t}^{-\infty} d t^{\prime}\left\langle a_{-k}^{(i)}\left(t^{\prime}\right) a_{-k}^{(i) \dagger}(t)\right\rangle\left\langle a_{(k+q)}^{(i)}\left(t^{\prime}\right) a_{(k+q)}^{(i) \dagger}(t)\right\rangle \\
= & -\int_{-\infty}^{\infty} d t^{\prime}\left[g_{-k}^{(i) \mathrm{a}}\left(t^{\prime}, t\right) g_{(k+q)}^{(i)<}\left(t^{\prime}, t\right)+g_{-k}^{(i)>}\left(t^{\prime}, t\right) g_{(k+q)}^{(i) \mathrm{a}}\left(t^{\prime}, t\right)\right] .
\end{aligned}
$$

Using

$$
\begin{aligned}
& g_{k \omega}^{>}=-\left(1+n_{k}\right)\left(g_{k \omega}^{\mathrm{a}}-g_{k \omega}^{\mathrm{r}}\right), \\
& g_{k \omega}^{<}=-n_{k}\left(g_{k \omega}^{\mathrm{a}}-g_{k \omega}^{\mathrm{r}}\right),
\end{aligned}
$$

products of the Green's functions are calculated as (noting that the integration in $\omega$ is finite only for terms containing either retarded or advanced Green's functions, as the frequencies for the two are $\omega$ and $-\omega$ )

$$
\begin{aligned}
g_{k}^{(i) \mathrm{r}}\left(t, t^{\prime}\right) g_{-(k+q)}^{(i)>}\left(t, t^{\prime}\right)+g_{k}^{(i)<}\left(t, t^{\prime}\right) g_{-(k+q)}^{(i) \mathrm{r}}\left(t, t^{\prime}\right) & =\int \frac{d \omega}{2 \pi} \int \frac{d \Omega}{2 \pi} e^{i \Omega\left(t-t^{\prime}\right)}\left[g_{k, \omega}^{(i) \mathrm{r}} g_{-(k+q),-(\omega+\Omega)}^{(i)>}+g_{k, \omega}^{(i)<} g_{-(k+q),-(\omega+\Omega)}^{(i) \mathrm{r}}\right] \\
& =\int \frac{d \omega}{2 \pi} \int \frac{d \Omega}{2 \pi} e^{i \Omega\left(t-t^{\prime}\right)}\left[\left(1+n_{-k-q}\right) g_{k, \omega}^{(i) \mathrm{r}} g_{-(k+q),-(\omega+\Omega)}^{(i) \mathrm{r}}+n_{k} g_{k, \omega}^{(i) \mathrm{r}} g_{-(k+q),-(\omega+\Omega)}^{(i) \mathrm{r}}\right]
\end{aligned}
$$




$$
\begin{aligned}
= & i \int \frac{d \Omega}{2 \pi} e^{i \Omega\left(t-t^{\prime}\right)} \frac{1+n_{k}+n_{-(k+q)}}{\omega_{-(k+q)}^{(i)}+\omega_{k}^{(i)}+\Omega-2 i \eta}, \\
g_{-k}^{(i) \mathrm{a}}\left(t^{\prime}, t\right) g_{(k+q)}^{(i)<}\left(t^{\prime}, t\right)+g_{-k}^{(i)>}\left(t^{\prime}, t\right) g_{(k+q)}^{(i) \mathrm{a}}\left(t^{\prime}, t\right)= & \int \frac{d \omega}{2 \pi} \int \frac{d \Omega}{2 \pi} e^{i \Omega\left(t-t^{\prime}\right)}\left[g_{-k,-\omega}^{(i) \mathrm{a}} g_{(k+q),(\omega+\Omega)}^{(i)<}+g_{-k,-\omega}^{(i)>} g_{(k+q),(\omega+\Omega)}^{(i) \mathrm{a}}\right] \\
= & \int \frac{d \omega}{2 \pi} \int \frac{d \Omega}{2 \pi} e^{i \Omega\left(t-t^{\prime}\right)}(-)\left(n_{k+q}+1+n_{-k}\right) \\
& \times\left[g_{-k,-\omega}^{(i) \mathrm{a}} g_{(k+q),(\omega+\Omega)}^{(i) \mathrm{a}}+g_{-k,-\omega}^{(i) \mathrm{a}} g_{(k+q),(\omega+\Omega)}^{(i) \mathrm{a}}\right] \\
= & i \int \frac{d \Omega}{2 \pi} e^{i \Omega\left(t-t^{\prime}\right)} \frac{1+n_{-k}+n_{(k+q)}}{\omega_{(k+q)}^{(i)}+\omega_{-k}^{(i)}-\Omega+2 i \eta} .
\end{aligned}
$$

The result of the amplitudes of the two-magnon annihilation and creation is

$$
\begin{aligned}
&\left\langle a_{-(k+q)}^{(i)} a_{k}^{(i)}\right\rangle(t)=\frac{K g}{a} \int \frac{d \Omega}{2 \pi} e^{i \Omega t} \int_{-\infty}^{\infty} d t^{\prime} e^{-i \Omega t^{\prime}} e^{i q X\left(t^{\prime}\right)} \lambda\left(t^{\prime}\right) \frac{W_{-q}}{\sqrt{\omega_{k}^{(i)} \omega_{k+q}^{(i)}}} \frac{1+n_{k}+n_{-(k+q)}^{(i)}}{\omega_{-(k+q)}+\omega_{k}^{(i)}+\Omega-2 i \eta} \\
&\left\langle a_{(k+q)}^{(i) \dagger} a_{-k}^{(i) \dagger}\right\rangle(t)=\frac{K g}{a} \int \frac{d \Omega}{2 \pi} e^{i \Omega t} \int_{-\infty}^{\infty} d t^{\prime} e^{-i \Omega t^{\prime}} e^{i q X\left(t^{\prime}\right)} \lambda\left(t^{\prime}\right) \frac{W_{-q}}{\sqrt{\omega_{k}^{(i)} \omega_{k+q}^{(i)}}} \frac{1+n_{-k}+n_{(k+q)}}{\omega_{(k+q)}^{(i)}+\omega_{-k}^{(i)}-\Omega+2 i \eta} .
\end{aligned}
$$

Changing $k \rightarrow-k$,

$$
\left\langle a_{(-k+q)}^{(i) \dagger} a_{k}^{(i) \dagger}\right\rangle(t)=-i \frac{K g}{a} \int \frac{d \Omega}{2 \pi} e^{i \Omega t} \int_{-\infty}^{\infty} d t^{\prime} e^{-i \Omega t^{\prime}} e^{i q X\left(t^{\prime}\right)} \lambda\left(t^{\prime}\right) \frac{W_{-q}}{\sqrt{\omega_{k}^{(i)} \omega_{-k+q}^{(i)}}} \frac{1+n_{-k}+n_{(k+q)}}{\omega_{(-k+q)}^{(i)}+\omega_{k}^{(i)}-\Omega+2 i \eta} .
$$

Here $\hbar q$ and $\hbar \Omega$ are the momentum and energy given to the spin waves from the wall, respectively.

We define response functions for scattering and emission as

$$
\begin{aligned}
\Pi_{k, q, \Omega} & \equiv-\frac{W_{-q}}{\sqrt{\omega_{k}^{(i)} \omega_{k+q}^{(i)}}} \frac{n_{k+q}-n_{k}}{\omega_{k+q}-\omega_{k}-\Omega+2 i \eta}, \\
\Gamma_{k, q, \Omega} & \equiv \frac{W_{-q}}{\sqrt{\omega_{k}^{(i)} \omega_{-k+q}^{(i)}}} \frac{1+n_{-k}+n_{(k+q)}}{\omega_{(-k+q)}^{(i)}+\omega_{k}^{(i)}-\Omega+2 i \eta},
\end{aligned}
$$

so that

$$
\begin{aligned}
\left\langle a_{k+q}^{(i) \dagger} a_{k}^{(i)}\right\rangle(t) & =\frac{K g}{a} \int \frac{d \Omega}{2 \pi} e^{i \Omega t} \int_{-\infty}^{\infty} d t^{\prime} e^{-i \Omega t^{\prime}} e^{i q X\left(t^{\prime}\right)} \lambda\left(t^{\prime}\right) \sum_{k} \Pi_{k, q, \Omega}, \\
\left\langle a_{-k+q}^{(i) \dagger} a_{k}^{(i) \dagger}\right\rangle(t) & =\frac{K g}{a} \int \frac{d \Omega}{2 \pi} e^{i \Omega t} \int_{-\infty}^{\infty} d t^{\prime} e^{-i \Omega t^{\prime}} e^{i q X\left(t^{\prime}\right)} \lambda\left(t^{\prime}\right) \sum_{k} \Gamma_{k, q, \Omega},
\end{aligned}
$$

and

$$
\left\langle a_{-k+q}^{(i)} a_{k}^{(i)}\right\rangle(t)=\frac{K g}{a} \int \frac{d \Omega}{2 \pi} e^{i \Omega t} \int_{-\infty}^{\infty} d t^{\prime} e^{-i \Omega t^{\prime}} e^{i q X\left(t^{\prime}\right)} \lambda\left(t^{\prime}\right) \sum_{k} \Gamma_{k,-q,-\Omega}^{*}
$$

for the absorption. $\Pi_{k, q, \Omega}$ represents an ordinary particle-hole propagation, while $\Gamma_{k, q, \Omega}$ is an anomalous particle-particle (or hole-hole) propagation.

The correlation functions after the summation over $k$ have the following properties (we use $W_{-q}=W_{q}$ and $\omega_{-k}=\omega_{k}$ ):

$$
\begin{aligned}
\sum_{k} \Pi_{k,-q, \Omega} & =-\sum_{k^{\prime}=-k} \frac{W_{-q}}{\sqrt{\omega_{k^{\prime}}^{(i)} \omega_{-k^{\prime}-q}^{(i)}}} \frac{n_{-k^{\prime}-q}-n_{k^{\prime}}}{\omega_{-k^{\prime}-q}-\omega_{k^{\prime}}-\Omega+2 i \eta}=\sum_{k} \Pi_{k, q, \Omega}, \\
\sum_{k} \Gamma_{k,-q, \Omega} & =\sum_{k^{\prime}=-k} \frac{W_{-q}}{\sqrt{\omega_{k^{\prime}}^{(i)} \omega_{k^{\prime}-q}^{(i)}}} \frac{1+n_{k^{\prime}}+n_{\left(k^{\prime}-q\right)}^{(i)}}{\omega_{\left(k^{\prime}-q\right)}+\omega_{k^{\prime}}^{(i)}-\Omega+2 i \eta}=\sum_{k} \Gamma_{k, q, \Omega} .
\end{aligned}
$$


Namely, both are even in $q$ if $\Omega$ is independent of $q$. Later we consider the case of a motion with a constant velocity $V_{\mathrm{w}}$, and in that case, $\Omega=q V_{\mathrm{w}}$ is imposed. In this case, the symmetry is modified as

$$
\sum_{k} \Pi_{k,-q, \Omega=-q V_{\mathrm{w}}}=-\sum_{k^{\prime}=k-q} \frac{W_{-q}}{\sqrt{\omega_{k^{\prime}}^{(i)} \omega_{k^{\prime}+q}^{(i)}}} \frac{n_{k^{\prime}+q}-n_{k^{\prime}}}{\omega_{k^{\prime}+q}-\omega_{k^{\prime}}-q V_{\mathrm{w}}-2 i \eta}=\Pi_{q, \Omega=q V_{\mathrm{w}}}^{*} .
$$

Therefore,

$$
\begin{aligned}
& \operatorname{Re}\left[\sum_{k} \Pi_{k,-q, \Omega=-q V_{\mathrm{w}}}\right]=\operatorname{Re}\left[\sum_{k} \Pi_{k, q, \Omega=q V_{\mathrm{w}}}^{*}\right] \\
& \operatorname{Im}\left[\sum_{k} \Pi_{k,-q, \Omega=-q V_{\mathrm{w}}}\right]=-\operatorname{Im}\left[\sum_{k} \Pi_{k, q, \Omega=q V_{\mathrm{w}}}^{*}\right] .
\end{aligned}
$$

The emission response function is

$$
\sum_{k} \Gamma_{k,-q, \Omega=-q V_{\mathrm{w}}}=\sum_{k} \Gamma_{k, q, \Omega=-q V_{\mathrm{w}}}
$$

and have both even and odd contributions of $q$ when $V_{\mathrm{w}} \neq 0$.

\section{APPENDIX D: LORENTZ BOOST OF RESPONSE FUNCTION}

In the case of normal relativistic dispersion, the system has a Lorentz invariance. The spin wave emission and scattering from a moving wall is understood from the Lorentz boost of the response function. The real transition (imaginary part) contributions of the response functions in $q-\Omega$ space are

$$
\begin{aligned}
\Pi_{q, \Omega} & =-\frac{1}{V} \sum_{k} \frac{W_{-q}}{\sqrt{\omega_{k}^{(i)} \omega_{k+q}^{(i)}}} \frac{\eta\left(n_{k+q}-n_{\boldsymbol{k}}\right)}{\left(\omega_{\boldsymbol{k}+\boldsymbol{q}}^{(i)}-\omega_{\boldsymbol{k}}^{(i)}-\Omega\right)^{2}+\eta^{2}}, \\
\Gamma_{q, \Omega} & =\frac{1}{V} \sum_{k} \frac{W_{-q}}{\sqrt{\omega_{k}^{(i)} \omega_{-k+q}^{(i)}}} \frac{\eta\left(1+n_{k}+n_{-k+q}\right)}{\left(\omega_{-k+q}^{(i)}+\omega_{k}^{(i)}-\Omega\right)^{2}+\eta^{2}} .
\end{aligned}
$$

The response in the boosted frame with velocity $v$ is represented by changing variables as

$$
u \equiv \frac{x-v t}{\gamma}, \quad s \equiv \frac{t-v x / c^{2}}{\gamma},
$$

where $\gamma \equiv \sqrt{1-(v / c)^{2}}$. The response in the boosted frame is given by (the same for $\Gamma$ )

$$
\begin{aligned}
\sum_{q} \int \frac{d \Omega}{2 \pi} e^{-i q x} e^{i \Omega t} \Pi_{q, \Omega} \\
=\sum_{q} \int \frac{d \Omega}{2 \pi} e^{-i u(q-v \Omega) / \gamma} e^{i s(\Omega-v q) / \gamma} \Pi_{q, \Omega} \\
=\sum_{q} \int \frac{d \Omega}{2 \pi} e^{-i q u} e^{i \Omega s} \Pi_{(q+v \Omega) / \gamma,(\Omega+v q) / \gamma} .
\end{aligned}
$$

The response functions are distorted due to the mixing of $q$ and $\Omega$, as shown in Fig. 13. The response functions from the moving wall agree with the boost of the static $(\Omega=0)$ rest-frame one $\Pi_{q / \gamma, v q / \gamma}$. The boost transformation clearly indicates the appearance of a peak at finite $q$, which leads to a significant emission discussed in the main text.

\section{APPENDIX E: RELATION TO $\xi$ REPRESENTATION OF REFERENCE [8]}

To describe fluctuation around a domain wall, parametrization in terms of the $\xi$ variable, defined as

$$
\xi \equiv e^{i \phi} \tan \frac{\theta}{2}
$$

has been used in Refs. [8,20]. For the case of a domain wall centered at $x=X$ with the angle of plane $\phi_{0}, \xi$ is

$$
\xi=e^{-u+i \phi_{0}+\eta},
$$

where $u=\frac{x-X}{\lambda}$, and $\eta$ represents the spin wave. This representation is equivalent to the rotated-frame representation in the present paper. In fact, $\eta \equiv \eta_{\mathrm{R}}+i \eta_{\mathrm{I}}$ and $\boldsymbol{\varphi}=\left(\varphi_{x}, \varphi_{y}\right)$ are related as $\tilde{\eta}_{\mathrm{R}}=\frac{1}{2} \varphi_{x}$ and $\tilde{\eta}_{\mathrm{I}}=\frac{1}{2} \varphi_{y}$, where $\tilde{\eta} \equiv \frac{1}{2 \cosh u} \eta$.
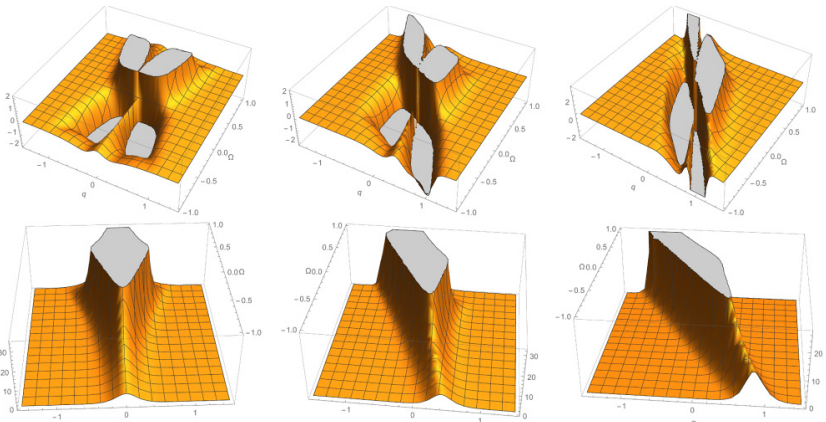

FIG. 13. The Lorentz boost effects on the imaginary parts of the normal (upper row) and the anomalous (lower row) response functions, $\operatorname{Im}\left[\Pi_{(q+v \Omega) / \gamma,(\Omega+v q) / \gamma}\right]$ and $\operatorname{Im}\left[\Gamma_{(q+v \Omega) / \gamma,(\Omega+v q) / \gamma}\right]$, respectively, for the relativistic dispersion at $v / c=0,0.4,0.8$ from the left to the right. 
[1] M. Petev, N. Westerberg, D. Moss, E. Rubino, C. Rimoldi, S. L. Cacciatori, F. Belgiorno, and D. Faccio, Blackbody Emission from Light Interacting with an Effective Moving Dispersive Medium, Phys. Rev. Lett. 111, 043902 (2013).

[2] L. Ostrovskii, Some general relations for waves at the moving boundary between two media, Zh. Eksp. Teor. Fiz. 61, 551 (1972) [Sov. Phys. JETP 34, 293 (1972)].

[3] F. D. M. Haldane, Nonlinear Field Theory of Large-Spin Heisenberg Antiferromagnets: Semiclassically Quantized Solitons of the One-Dimensional Easy-Axis Néel State, Phys. Rev. Lett. 50, 1153 (1983).

[4] T. Shiino, S.-H. Oh, P. M. Haney, S.-W. Lee, G. Go, B.-G. Park, and K.-J. Lee, Antiferromagnetic Domain Wall Motion Driven by Spin-Orbit Torques, Phys. Rev. Lett. 117, 087203 (2016).

[5] D. Bouzidi and H. Suhl, Motion of a Bloch Domain Wall, Phys. Rev. Lett. 65, 2587 (1990).

[6] Y. Le Maho, J.-V. Kim, and G. Tatara, Spin-wave contributions to current-induced domain wall dynamics, Phys. Rev. B 79, 174404 (2009).

[7] S. K. Kim, O. Tchernyshyov, V. Galitski, and Y. Tserkovnyak, Magnon-induced non-Markovian friction of a domain wall in a ferromagnet, Phys. Rev. B 97, 174433 (2018).

[8] G. Tatara and R. M. Otxoa de Zuazola, Collective coordinate study of spin-wave emission from a dynamic domain wall, Phys. Rev. B 101, 224425 (2020).

[9] J. C. Slonczewski, Dynamics of magnetic domain walls, Int. J. Magn. 2, 85 (1972).

[10] G. Tatara and H. Kohno, Theory of Current-Driven Domain Wall Motion: Spin Transfer versus Momentum Transfer, Phys. Rev. Lett. 92, 086601 (2004).

[11] X. S. Wang, P. Yan, Y. H. Shen, G. E. W. Bauer, and X. R. Wang, Domain Wall Propagation Through Spin Wave Emission, Phys. Rev. Lett. 109, 167209 (2012).

[12] G. Tatara and C. O. Pauyac, Theory of spin transport through an antiferromagnetic insulator, Phys. Rev. B 99, 180405(R) (2019).

[13] E. Rubino, A. Lotti, F. Belgiorno, S. L. Cacciatori, A. Couairon, U. Leonhardt, and D. Faccio, Soliton-induced relativisticscattering and amplification, Sci. Rep. 2, 932 (2012).

[14] E. G. Tveten, A. Qaiumzadeh, and A. Brataas, Antiferromagnetic Domain Wall Motion Induced by Spin Waves, Phys. Rev. Lett. 112, 147204 (2014).
[15] O. Gomonay, T. Jungwirth, and J. Sinova, High Antiferromagnetic Domain Wall Velocity Induced by Néel Spin-Orbit Torques, Phys. Rev. Lett. 117, 017202 (2016).

[16] H. Yang, H. Y. Yuan, M. Yan, H. W. Zhang, and P. Yan, Atomic antiferromagnetic domain wall propagation beyond the relativistic limit, Phys. Rev. B 100, 024407 (2019).

[17] R. M. Otxoa, P. E. Roy, R. Rama-Eiroa, J. Godinho, K. Y. Guslienko, and J. Wunderlich, Walker-like Domain Wall breakdown in layered antiferromagnets driven by staggered spin-orbit fields, Commun. Phys. 3, 190 (2020).

[18] J. Schwinger, On gauge invariance and vacuum polarization, Phys. Rev. 82, 664 (1951).

[19] D. Allor, T. D. Cohen, and D. A. McGady, Schwinger mechanism and graphene, Phys. Rev. D 78, 096009 (2008).

[20] G. Tatara, H. Kohno, and J. Shibata, Microscopic approach to current-driven domain wall dynamics, Phys. Rep. 468, 213 (2008).

[21] One could introduce other coordinates such as the angle of the wall plane and thickness, as in the case of a ferromagnet [8], but they are essentially decoupled from the $X$ dynamics, except for the case where the driving field mixes them as discussed in Ref. [4].

[22] S. M. Rezende, A. Azevedo, and R. L. Rodríguez-Suárez, Introduction to antiferromagnetic magnons, J. Appl. Phys. 126, 151101 (2019).

[23] To be mathematically consistent, magnon dispersion is determined by the Lagrangian (1) and is correlated to the equation of motion of the domain wall. In reality, on the other hand, the dispersion is sensitive to details of lattice structure, while the collective behavior of a macroscopic wall is not. Considering a dispersion away from the relativistic limit would therefore be justified. Moreover, in real materials, the Lorentz invariance applies only as an approximation due to the existence of the lattice and dissipation.

[24] G. Tatara, Y.-W. Zhao, M. Muñoz, and N. García, Domain Wall Scattering Explains 300\% Magnetoconductance of Nanocontacts, Phys. Rev. Lett. 83, 2030 (1999).

[25] Z. Ebadi and B. Mirza, Entanglement generation by electric field background, Ann. Phys. 351, 363 (2014).

[26] G. Tatara, Effective gauge field theory of spintronics, Physica E 106, 208 (2019). 